\title{
On coupled-channel dynamics in the presence of anomalous thresholds
}

\author{
M. F. M. Lutz ${ }^{1,2}$ and C. L. Korpa ${ }^{3,4}$ \\ ${ }^{1}$ GSI Helmholtzzentrum für Schwerionenforschung GmbH, Planckstraße 1, 64291 Darmstadt, Germany \\ ${ }^{2}$ Technische Universität Darmstadt, D-64289 Darmstadt, Germany \\ ${ }^{3}$ Department of Theoretical Physics, University of Pécs, Ifjúság útja 6, 7624 Pécs, Hungary \\ ${ }^{4}$ ExtreMe Matter Institute EMMI, GSI Helmholtzzentrum für Schwerionenforschung, \\ Planckstrasse 1, 64291 Darmstadt, Germany
}

(Received 24 August 2018; published 5 October 2018)

\begin{abstract}
We explore a general framework to treat coupled-channel systems in the presence of overlapping left- and right-hand cuts as well as anomalous thresholds. Such systems are studied in terms of a generalized potential, where we exploit the known analytic structure of $t$ - and $u$-channel forces as the exchange masses approach their physical values. Given an approximate generalized potential the coupled-channel reaction amplitudes are defined in terms of nonlinear systems of integral equations. For large exchange masses, where there are no anomalous thresholds present, conventional $N / D$ methods are applicable to derive numerical solutions to the latter. At a formal level a generalization to the anomalous case is readily formulated by use of suitable contour integrations with amplitudes to be evaluated at complex energies. However, it is a considerable challenge to find numerical solutions to anomalous systems set up on a set of complex contours. By suitable deformations of left-hand and right-hand cut lines we manage to establish a framework of linear integral equations defined for real energies. Explicit expressions are derived for the driving terms that hold for an arbitrary number of channels. Our approach is illustrated in terms of schematic three-channel systems. It is demonstrated that despite the presence of anomalous thresholds the scattering amplitude can be represented in terms of three phase shifts and three inelasticity parameters, as one would expect from the coupled-channel unitarity condition.
\end{abstract}

DOI: 10.1103/PhysRevD.98.076003

\section{INTRODUCTION}

A reliable and systematic treatment of coupledchannel systems subject to strong interactions is still one of the remaining fundamental challenges of modern physics. So far effective field theory approaches with hadronic degrees of freedom (d.o.f.) that reflect QCD properties are established only for particular corners of the strong interaction world. At energies where QCD forms bound states or resonances there is a significant lack of profound theory that connects to experimental data directly. Despite the tremendous efforts and successes of experimental accelerator facilities and emerging lattice gauge theory simulations there is a significant gap in what theory can accomplish and experimental groups would need to be properly guided in new searches for exotic matter [1-4].

Published by the American Physical Society under the terms of the Creative Commons Attribution 4.0 International license. Further distribution of this work must maintain attribution to the author(s) and the published article's title, journal citation, and DOI. Funded by SCOAP ${ }^{3}$.
To unfold the underlying physics of this nonperturbative domain of QCD novel approaches are required that combine the power of coupled-channel unitarity together with the microcausality condition for hadronic d.o.f. [5-24]. While such frameworks exist for coupledchannel interactions that are dominated by short-range forces matters become significantly more challenging in the presence of $t$ - or $u$-channel long-range forces [20-28]. In particular coupled-channel systems involving the nonet of vector mesons with $J^{P}=1^{-}$or the baryon decuplet states with $J^{P}=\frac{3}{2}{ }^{+}$can only be studied with significant results after such a framework has been developed. The latter play a crucial role in the hadrogenesis conjecture that expects the low-lying resonance spectrum of QCD with up, down and strange quarks only, to be generated by final-state interactions of the lowest $\mathrm{SU}(3)$ flavor multiplets with $J^{P}=0^{-}, 1^{-}$ and $J^{P}=\frac{1}{2}{ }^{+}, \frac{3}{2}{ }^{+}[13,14,16,17,29-35]$. The coupledchannel interaction of such d.o.f. leads to a plethora of subtle effects, like numerous anomalous thresholds [36-38].

The physical relevance of anomalous threshold effects has been discussed recently in Refs. [39-44]. To the best of 
our knowledge there is no established approach available that can treat such phenomena in coupled-channel systems reliably. In the previous works which attempted to deal with such systems the strategy was to perform an analytic continuation of an $N / D$ ansatz for the reaction amplitudes in the external mass parameters so as to smoothly connect a normal system to an anomalous system. This was studied for two-channel systems only [37,38]. Even there the first study of Ball, Frazer and Nauenberg [37] was determined by the later work of Greben and Kok to be incorrect [38]. So far we have not been able to track any numerical implementation of either of the two schemes [37,38]. Following this strategy an extension to a truly multichannel system appears prohibitively cumbersome.

A powerful framework to study coupled-channel systems is based on the concept of a generalized potential. A partial-wave scattering amplitude $T_{a b}(s)$ where the channel indices $a$ and $b$ stand for the final and the initial state respectively is decomposed into contributions from leftand right-hand cuts where all left-hand cut contributions are collected into the generalized potential $U_{a b}(s)$. For a given generalized potential the right-hand cuts are implied by the nonlinear integral equation

$$
T_{a b}(s)=U_{a b}(s)+\sum_{c, d} \int \frac{d \bar{s}}{\pi} \frac{s-\mu^{2}}{\bar{s}-\mu^{2}} \frac{T_{a c}^{\dagger}(\bar{s}) \rho_{c d}(\bar{s}) T_{d b}(\bar{s})}{\bar{s}-s},
$$

where $\rho_{c d}(s)$ is a channel-dependent phase-space function and all integrals are for $\bar{s}$ on the real axis. By construction any solution of Eq. (1) does satisfy the coupledchannel $s$-channel unitarity condition for normal systems. Typically, the matching scale $\mu^{2}$ in Eq. (1) is to be chosen in a kinematical region where the reaction amplitude can be computed in perturbation theory $[13,17,20]$. For normal systems the nonlinear and coupled set of equations (1) can be solved by an $N / D$ ansatz [20,21,45-52]. Although the general framework has been known since the 1960 s, it was only recently successfully integrated into an effective field theory approach based on the chiral Lagrangian [20-26]. As it stands Eq. (1) breaks down once a coupled-channel system involves unstable particles or anomalous thresholds arise. In this work we will construct a suitable adaptation that overcomes this gap.

Our formal developments will be illustrated by a schematic three-channel model where explicit numerical results for key quantities will be presented along the way. In Secs. II and III we discuss the analytic structure of the coupled-channel reaction amplitudes and propose an efficient deformation of the left- and right-hand cut lines such that anomalous systems can be dealt with. In addition our schematic model is specified. In Sec. IV an ansatz for the
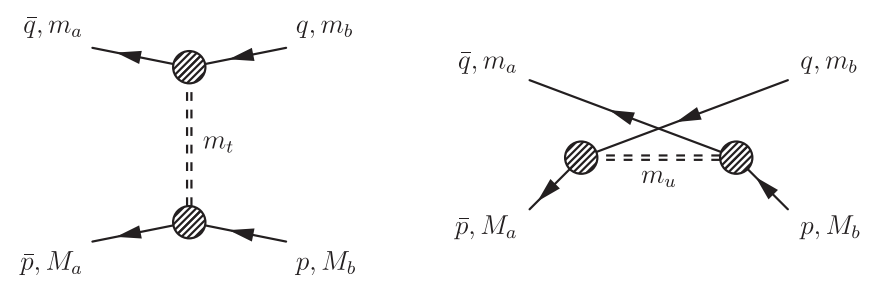

FIG. 1. Generic $t$ - and $u$-channel exchange processes.

solution of the nonlinear integral equations on the complex contour lines is derived. In the next step in Sec. V we consider the limit where the complex contour lines are deformed back towards the real energy line. The main formal result of our work is derived in Sec. VI, where a set of linear integral equations for anomalous systems that is suitable for numerical implementations is established. We close with a summary and outlook in Sec. VII.

\section{ANALYTIC STRUCTURE OF PARTIAL-WAVE SCATTERING AMPLITUDES}

In a first step we will recall a spectral representation for a generic $t$-channel and $u$-channel term as shown in Fig. 1. In our previous work [28] we established the following general form:

$$
\begin{aligned}
U_{a b}^{(\mathrm{t}-\mathrm{ch} .)}(s) & =\sum_{i= \pm} \int_{-\infty}^{\infty} \frac{d m^{2}}{\pi} \frac{\varrho_{i, a b}^{(t)}\left(m^{2}, m_{t}^{2}\right)}{s-c_{i, a b}^{(t)}\left(m^{2}\right)}\left(\frac{d}{d m^{2}} c_{i, a b}^{(t)}\left(m^{2}\right)\right), \\
U_{a b}^{(\mathrm{u}-\mathrm{ch} .)}(s) & =\sum_{i= \pm} \int_{-\infty}^{\infty} \frac{d m^{2}}{\pi} \frac{\varrho_{i, a b}^{(u)}\left(m^{2}, m_{u}^{2}\right)}{s-c_{i, a b}^{(u)}\left(m^{2}\right)}\left(\frac{d}{d m^{2}} c_{i, a b}^{(u)}\left(m^{2}\right)\right),
\end{aligned}
$$

with properly constructed spectral weights $\varrho_{ \pm, a b}^{(t)}\left(m^{2}, m_{t}^{2}\right)$ and $\varrho_{ \pm, a b}^{(u)}\left(m^{2}, m_{u}^{2}\right)$. The contour functions $c_{ \pm, a b}^{(t)}\left(m^{2}\right)$ and $c_{ \pm, a b}^{(u)}\left(m^{2}\right)$ depend on the masses of initial and final particles of the given reaction $a b$ for which we use the convenient notation

$$
q^{2}=m_{b}^{2}, \quad \bar{q}^{2}=m_{a}^{2}, \quad p^{2}=M_{b}^{2}, \quad \bar{p}^{2}=M_{a}^{2} .
$$

While the derivation of the spectral weights $\varrho_{ \pm, a b}^{(t)}\left(m^{2}, t\right)$ and $\varrho_{ \pm, a b}^{(u)}\left(m^{2}, u\right)$ is quite cumbersome the identification of the contour functions $c_{ \pm}^{(t)}\left(m^{2}\right)$ and $c_{ \pm}^{(u)}\left(m^{2}\right)$ is straightforward. Owing to the Landau equations any possible branch point of a partial-wave amplitude must be associated with an end-point singularity of the partial-wave projection integral that involves some Legendre polynomials in $\cos \theta$ of the scattering angle $\theta$. This leads to the wellknown result 


$$
\begin{aligned}
c_{ \pm, a b}^{(u)}\left(m^{2}\right)= & \frac{1}{2}\left(M_{a}^{2}+m_{a}^{2}+M_{b}^{2}+m_{b}^{2}-m^{2}\right)+\frac{M_{a}^{2}-m_{b}^{2}}{\sqrt{2} m} \frac{M_{b}^{2}-m_{a}^{2}}{\sqrt{2} m} \\
& \pm \frac{m^{2}}{2} \sqrt{\left(1-2 \frac{M_{a}^{2}+m_{b}^{2}}{m^{2}}+\frac{\left(M_{a}^{2}-m_{b}^{2}\right)^{2}}{m^{4}}\right)\left(1-2 \frac{M_{b}^{2}+m_{a}^{2}}{m^{2}}+\frac{\left(M_{b}^{2}-m_{a}^{2}\right)^{2}}{m^{4}}\right),} \\
c_{ \pm, a b}^{(t)}\left(m^{2}\right)= & \frac{1}{2}\left(M_{a}^{2}+m_{a}^{2}+M_{b}^{2}+m_{b}^{2}-m^{2}\right)-\frac{M_{a}^{2}-M_{b}^{2}}{\sqrt{2} m} \frac{m_{a}^{2}-m_{b}^{2}}{\sqrt{2} m} \\
& \pm \frac{m^{2}}{2} \sqrt{\left(1-2 \frac{M_{a}^{2}+M_{b}^{2}}{m^{2}}+\frac{\left(M_{a}^{2}-M_{b}^{2}\right)^{2}}{m^{4}}\right)\left(1-2 \frac{m_{a}^{2}+m_{b}^{2}}{m^{2}}+\frac{\left(m_{a}^{2}-m_{b}^{2}\right)^{2}}{m^{4}}\right)} .
\end{aligned}
$$

An anomalous system arises if either of the spectral weights $\varrho_{i}^{(t)}\left(m^{2}, m_{t}^{2}\right)$ or $\varrho_{i}^{(u)}\left(m^{2}, m_{u}^{2}\right)$ is nonvanishing at an exchange mass $m$ where the associated contour $c_{i}^{(t)}\left(m^{2}\right)$ or $c_{i}^{(u)}\left(m^{2}\right)$ approaches any of the thresholds or pseudothresholds of the given reaction $a b$. In this case the decomposition (1) breaks down: an anomalous threshold behavior is encountered. The latter is characterized by a particular branch point $\mu_{a b}^{A}$ of the partial-wave amplitude

$$
\mu_{a b}^{A}<\operatorname{Min}\left\{m_{a}+M_{a}, m_{b}+M_{b}\right\},
$$

that is associated with the given amplitude $a b$. For simplicity of the presentation we consider in Eq. (5) an anomalous threshold behavior at a normal threshold point only. A similar phenomenon may occur at a pseudothreshold. Also the case $\mu_{a b}^{A}>\operatorname{Max}\left\{m_{a}+M_{a}, m_{b}+M_{b}\right\}$ is not excluded in general. For both cases, our approach can be adapted in a straightforward manner. However, the fact that an anomalous threshold arises for a diagonal reaction with $a=b$ has been excluded from general considerations.

In the previous works an analytic continuation in the external mass parameters was attempted so as to smoothly connect a normal two-channel system to an anomalous two-channel system $[37,38]$. After all using this method Mandelstam gave a transparent presentation of the anomalous threshold phenomenon in a typical one-loop diagram [36]. However, we feel that an application of this method directly to multichannel reaction amplitudes, as attempted in Refs. [37,38], appears futile due to the proliferation of branch points and cut structures that need to be properly deformed and followed up in various limits.

We argue that there is a significantly more transparent path to arrive at a framework that is capable of treating coupled-channel systems in the presence of anomalous thresholds. Given a multichannel system various anomalous thresholds may appear in different reactions. Our starting point, is based directly on the general representation (2), which already established a suitable analytic continuation of the tree-level potential terms valid for arbitrary exchange masses. As was emphasized in our previous work [28] the reason why a one-loop diagram develops an anomalous threshold can be read off easily from its driving tree-level potential terms.

We need to be somewhat more specific regarding the generic form of the generalized potential. The tree-level potential takes the generic form

$$
U_{a b}^{\mathrm{tree}-\mathrm{level}}(s)=\int_{L_{a b}} \frac{d \bar{s}}{\pi} \frac{\rho_{a b}^{U}(\bar{s})}{\bar{s}-s},
$$

where the left-hand contour, $L_{a b}$, is a union of contours required for the spectral representation of a general $t$ - or $u$ channel exchange process as in Eq. (2). In order to achieve our goal it is instrumental to separate the left-hand contour into a normal and an anomalous part

$$
L_{a b}=\Delta L_{a b} \cup A_{a b},
$$

where the part $A_{a b}$ starts at the anomalous threshold $\left(\mu_{a b}^{A}\right)^{2}$ slightly above the real axis, extends to the normal threshold point $\operatorname{Min}\left\{\left(m_{a}+M_{a}\right)^{2},\left(m_{b}+M_{b}\right)^{2}\right\}$ and returns at $\left(\mu_{a b}^{R}\right)^{2}$ to the anomalous one slightly below the real axis. This is illustrated in Fig. 2. Note that according to the general representation (2) the anomalous contour line would be on the real axis only. With Fig. 2 such a contour is deformed into the complex plane in a manner that leaves its contribution to the generalized potential unchanged for $s$ outside the area encircled by the dashed line. While the values for $\mu_{a b}^{A}$ are determined by the specifics of the coupled-channels interactions, there is some freedom in choosing the location of the return points $\mu_{a b}^{R}$. Given the

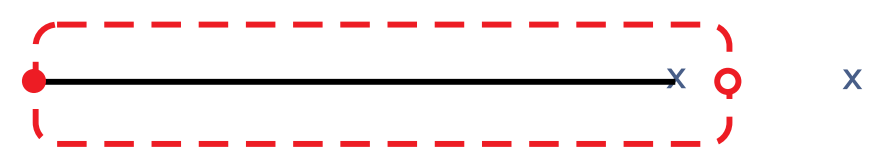

FIG. 2. Left-hand cut line of an anomalous contribution to the generalized potential. The two crosses show the locations of the threshold points at $\left(m_{a}+M_{a}\right)^{2}$ and $\left(m_{b}+M_{b}\right)^{2}$ where we assume $a<b$ without loss of generality. The filled and open red circles indicate the location of the anomalous threshold point $\left(\mu_{a b}^{A}\right)^{2}$ and a return point $\left(\mu_{a b}^{R}\right)^{2}$ respectively. 
analytic structure of the spectral weight $\rho_{a b}^{U}(\bar{s})$ the generalized potential $U_{a b}^{\text {tree-level }}(s)$ does not depend on the choice of the return point $\mu_{a b}^{R}$ at energies $s$ outside the dashed line of Fig. 2.

We point out a subtle issue such a contour separation is based on. Here we tacitly assumed that the anomalous threshold $\mu_{a b}^{A}$ is real. However, from the general representation (2) there is the possibility of a pair of complex-conjugate anomalous points $\mu_{a b}^{A \pm}$. In this case the associated contour lines have to be followed until both reach the common pseudothreshold point at $s=$ $\left(\mu_{a b}^{A}\right)^{2}=\operatorname{Max}\left\{\left(m_{a}-M_{a}\right)^{2},\left(m_{b}-M_{b}\right)^{2}\right\}$. The latter we take as the anomalous threshold value in our work. This can be justified since in this case the spectral weight $\rho_{a b}^{U}(\bar{s})$ can be shown to be analytic in $\epsilon$ to the left of the pseudothreshold point at $\bar{s}=\left(\mu_{a b}^{A}\right)^{2}-\epsilon$.

It is useful to identify an anomalous threshold value $\mu_{a}$ associated with a given channel $a$. To clarify the procedure we assume in the following a strict channel ordering according to the nominal threshold value, i.e., we insist on

$$
m_{a}+M_{a} \leq m_{a+1}+M_{a+1} \text { for all } a,
$$

where for later convenience we permit the equal sign for channels with the same two-particle states in different spin configurations only. We can now identify the desired anomalous threshold value. It is the minimum of all accessible anomalous branch points

$$
\mu_{a} \equiv \underbrace{\operatorname{Min}}_{b>a}\left\{\mu_{a b}^{A}, m_{a}+M_{a}\right\},
$$

where the value $\mu_{a b}^{A}$ gives the anomalous threshold value of the partial-wave amplitude $a b$. Note that it does not necessarily follow that the channel ordering (8) implies $\mu_{a+1} \geq \mu_{a}$. If such a channel crossing with $\mu_{a}>\mu_{b}$ for any pair ab with $a<b$ occurs, we will further move the point $\mu_{a}$ with $\mu_{a} \rightarrow \mu_{b}-\epsilon$ such that we ultimately arrive at a strict channel ordering

$$
\mu_{a} \leq \mu_{a+1} \text { for all } a,
$$

where again the equal sign is permitted for channels with the same two-particle states in different spin configurations only. It is useful to similarly streamline the plethora of return points. We choose universal return points $\hat{\mu}_{a}$ of the particular form

$$
\mu_{a b}^{R}=\hat{\mu}_{a}<m_{b}+M_{b} \text { for all } b>a,
$$

where we consider $a$ and $b$ with $\mu_{a b}^{A}<\left(m_{a}+M_{a}\right)^{2}$ only.

We can now introduce our anomalous contour $A_{a}$ that starts at $\mu_{a}^{2}$ slightly above the real axis, passes $\left(m_{a}+M_{a}\right)^{2}$ and returns at $\hat{\mu}_{a}^{2}$ to $\mu_{a}^{2}$ below the real axis. In turn we can now write
$U_{a b}(s)=\int_{\Delta L_{a b}} \frac{d \bar{s}}{\pi} \frac{\rho_{a b}^{\text {normal }}(\bar{s})}{\bar{s}-s}+\int_{A_{\operatorname{Min}(a, b)}} \frac{d \bar{s}}{\pi} \frac{\rho_{a b}^{\text {anomalous }}(\bar{s})}{\bar{s}-s}+\cdots$,

where we will exploit the fact that the first term in Eq. (12) is uncritical to the extent that it is analytic in the vicinity of any normal or anomalous threshold point. The challenging term is the second one, which is associated with a contour that entangles the normal threshold point $\operatorname{Min}\left\{\left(m_{a}+M_{b}\right)^{2},\left(m_{b}+M_{b}\right)^{2}\right\}$. The dots in Eq. (12) remind us that we have yet to discuss the generic form of contributions to the generalized potential from loop effects.

The key starting point is an adaptation of the nonlinear integral equation (1). The integral in Eq. (1) starts at the point where the phase-space matrix $\rho_{c d}(\bar{s})$ vanishes at $\bar{s}=$ $\left(m_{c}+M_{c}\right)^{2}=\left(m_{d}+M_{d}\right)^{2}$ and remains on the real axis going to infinity. In the anomalous case the integral must also start at $\bar{s}=\left(m_{c}+M_{c}\right)^{2}=\left(m_{d}+M_{d}\right)^{2}$ but will leave the real axis following suitable paths on higher Riemann sheets. The integral on the real axis has to be replaced by a contour integral. Eventually, somewhere on its way the contour path will touch the anomalous threshold at $\bar{s}=\mu_{c}^{2}$. Note that the case $c \neq d$ appears only for systems with nonvanishing spin.

This complication is a consequence of the anomalous threshold behavior of the generalized potential. If we wish to separate the left- from the right-hand cuts in the reaction amplitudes, it is necessary to apply suitable deformations of the cut lines. In the normal case the separation of left- from right-hand cuts is trivially implied by Eq. (1). The second term on the right-hand side has a cut on the real axis extending from $\bar{s}=\left(m_{1}+M_{1}\right)^{2}$ to infinity. This is the right-hand cut. All left-hand cuts sit in $U(s)$. As long as the cut lines of the generalized potential do not cross any of the right-hand cut lines on the real axis the nonlinear integral equation (1) is well defined and can be solved numerically using conventional methods. This is not the case if the generalized potential develops an anomalous threshold. Without an appropriate adaptation the associated left-hand cut lines would cross the right-hand cut lines. An avoidance is possible only if the integral on the real lines in Eq. (1) is replaced by contour integrals in the complex plane.

In Fig. 3 we illustrate the analytic structure of the reaction amplitudes defined with respect to suitably deformed leftand right-hand cut lines. The dashed red lines show the locations of the anomalous left-hand cuts. We suggest a deformation of the right-hand cut lines as illustrated by the solid lines in the figure. Now, none of the anomalous lefthand cut lines $A_{a}$ cross any of the right-hand contour lines $C_{a}$.

Before writing down the generalization of Eq. (1) we need some more notation. The reaction amplitudes $T_{a b}(s)$ need to be evaluated slightly below and above any of the many different contour lines, 


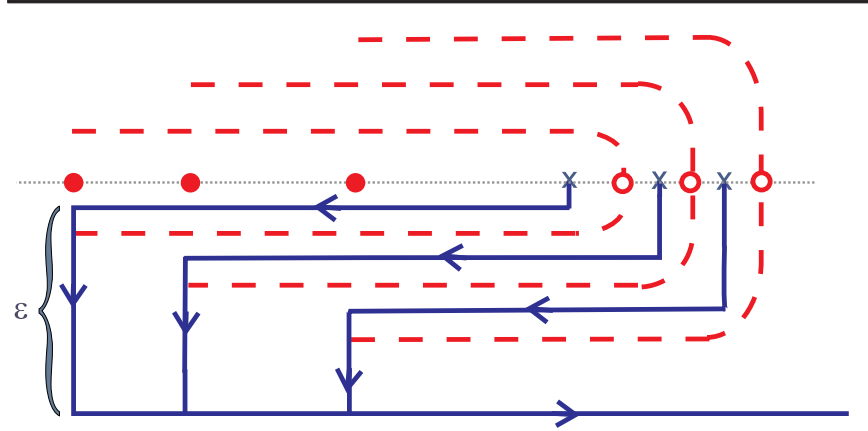

FIG. 3. Deformed left- and right-hand cut lines for the reaction amplitudes. The crosses show the locations of the threshold points at $\left(m_{a}+M_{a}\right)^{2}$. While the anomalous left-hand cut lines are shown with dashed red lines, the deformed right-hand cut lines are represented by blue solid lines. The filled and open red circles indicate the locations of the anomalous threshold points $\mu_{a}^{2}$ and the return points $\hat{\mu}_{a}^{2}$ respectively.

$$
T_{a b}^{c \pm}(\bar{s})=T_{a b}\left(s_{ \pm}\right) \quad \text { with } \quad \bar{s} \in C_{c},
$$

where the points $s_{+}$and $s_{-}$are an $\epsilon^{\prime}$ distance above and below the contour $C_{c}$ at $\bar{s}$ respectively. The value of $\epsilon^{\prime}$ is chosen smaller than the minimal distance of any of the horizontal lines in Fig. 3.

With this notation we can write down the desired generalization

$T_{a b}(s)=U_{a b}(s)+\sum_{c, d} \int_{C_{c}} \frac{d \bar{s} s-\mu^{2}}{\pi} \frac{T_{a c}^{c-}(\bar{s}) \rho_{c d}(\bar{s}) T_{d b}^{c+}(\bar{s})}{\bar{s}-\mu^{2}}$,

where $C_{c}=C_{d}$ holds by construction. We consider the phase-space matrix $\rho_{c d}(s)$ to be analytic on the real axis at
$s>\left(m_{c}+M_{c}\right)^{2}$. This implies that at $s<\left(m_{c}+M_{c}\right)^{2}$ the function $\rho_{c d}(s)$ has a cut line. Note that none of the contour paths $C_{c}$ cross any of the cut lines of the phasespace functions. It is emphasized that in the absence of an anomalous threshold in the generalized potential our generalization (14) reproduces the conventional expression (1).

The representation (14) appears deceivingly simple and ad hoc; however, we derived it by an analytic continuation in the $t$ - and $u$-channel exchange mass parameters. The starting point for this is provided by the general representation (2). First we assume all exchange particles to have a very large mass, so that all left-hand branch cuts are well separated from the right-hand branch cuts. Already at this stage we can deform the right-hand cuts from their conventional choice to take the form as indicated in Fig. 3 by the solid lines. This contour deformation cannot change the value of any of the coupled-channel reaction amplitudes $T_{a b}(s)$ at $\operatorname{Im} s>0$, simply because along the cut deformations the reaction amplitudes are analytic by assumption. Here we exploit the fact that in the limit of very large exchange masses all left-hand branch cuts are moved outside the figure. In a second step we follow the lefthand cuts as they move right in Fig. 3 as the exchange masses approach their physical values [36-38,48,52]. At the end we claim that they can be presented by the dashed lines in the figure. In turn we take Eq. (14) as a transparent starting point of our presentation.

Our numerical examples will rest on a minimal model as it is implied by schematic tree-level interactions. Along the formal derivations in the next few sections we will illustrate the important auxiliary quantities in terms of a schematic three-channel model specified with

$$
\begin{aligned}
\rho_{a b}(s) & =\frac{p_{a}^{+}(s)}{8 \pi \sqrt{s}} \delta_{a b}, \quad p_{a}^{ \pm}=\sqrt{ \pm\left(s-\left(m_{a}+M_{a}\right)^{2}\right) \frac{s-\left(m_{a}-M_{a}\right)^{2}}{4 s},} \\
\rho_{a b}^{A}(s) & =\frac{20 m_{\pi}^{2}}{p_{a}^{+}(s) i p_{b}^{-}(s)} \Theta\left[s-\mu_{a}^{2}\right] \Theta\left[\hat{\mu}_{a}^{2}-s\right]=\rho_{b a}^{A}(s) \quad \text { for } a<b, \\
U_{a b}^{\operatorname{model}}(s) & =\int_{A_{\operatorname{Min}(a, b)}} \frac{d \bar{s}}{\pi} \frac{\rho_{a b}^{A}(\bar{s})}{\bar{s}-s}, \\
m_{1} & =m_{2}=m_{3}=m_{\pi}, \quad \mu^{2}=20 m_{\pi}^{2}, \\
M_{1} & =4.2 m_{\pi}, \quad M_{2}=4.5 m_{\pi}, \quad M_{3}=4.8 m_{\pi}, \quad \hat{\mu}_{2}^{2}=32 m_{\pi}^{2} . \\
\mu_{1}^{2} & =25 m_{\pi}^{2}, \quad \hat{\mu}_{1}^{2}=28 m_{\pi}^{2}, \quad \mu_{2}^{2}=29 m_{\pi}^{2}, \quad \hat{\mu}_{2}
\end{aligned}
$$

In Fig. 4 we plot the tree-level generalized potential for two kinematical cases. The left-hand panel shows the nonvanishing elements with $s$ strictly on the real axis. Here the potentials are characterized by significant variations close to the anomalous threshold points with $s=\mu_{a}^{2}$ and at the return points with $s=\hat{\mu}_{a}^{2}$ and $\mu_{a}^{2}<\left(m_{a}+M_{a}\right)^{2}<\hat{\mu}_{a}^{2}$. The potentials are always real and smooth at the threshold points $s=\left(m_{a}+M_{a}\right)^{2}$. The right-hand panel shows the corresponding potentials evaluated at $s-i \epsilon$, just below the double-cut structures as illustrated in Fig. 2. In this case the potentials have an imaginary part and in addition are singular at the threshold points $s=\left(m_{a}+M_{a}\right)^{2}$. 


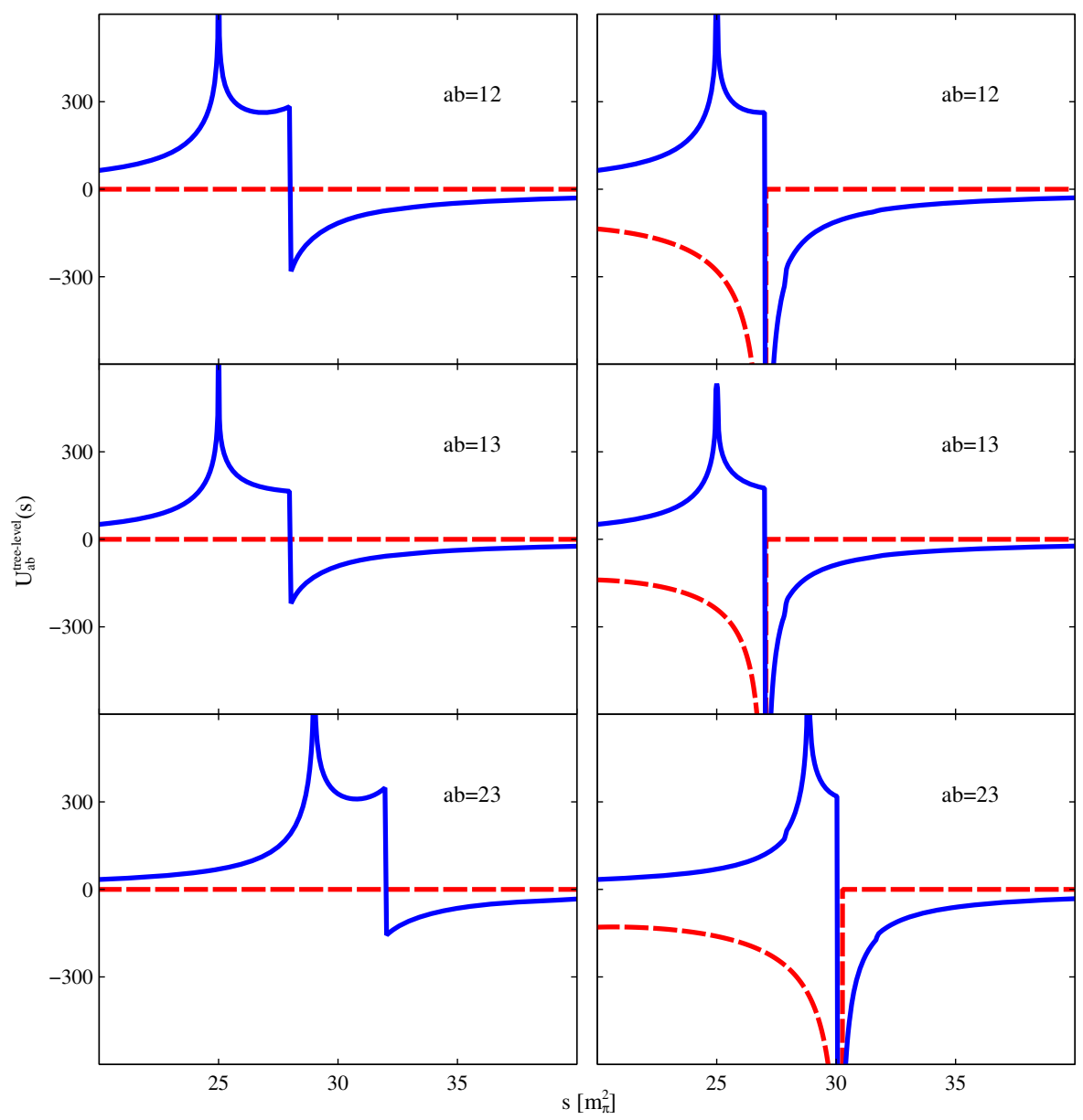

FIG. 4. The generalized potential $U_{a b}^{\text {tree-level }}(s)$ (left panels) and $U_{a b}^{\text {tree-level }}(s-i \epsilon)$ (right panels) in the schematic model as defined in Eq. (15). Only nonvanishing elements are considered. Real and imaginary parts are shown with solid blue and dashed red lines respectively.

In Fig. 5 we anticipate the usefulness of our formal developments and present the solution to the nonlinear integral equation (14) as implied by Eq. (15). It is noted that our results do not depend on the particular choices for the return points $\hat{\mu}_{a}$ in Eq. (15). The reaction amplitudes show significant and nontrivial structures that are implied by the presence of the anomalous threshold effects in the generalized potential. While the amplitudes at subthreshold energies show various singular structures, they are smooth and well behaved in the physical region. We emphasize that the subthreshold structures are not driven exclusively by the contribution from the generalized potential as shown in Fig. 4. There are in addition significant structures generated by the right-hand cut contributions. To the best of our knowledge with Fig. 5 we encounter the first numerical solution of such an anomalous three-channel system in the published literature.

\section{ANOMALOUS THRESHOLDS AND COUPLED-CHANNEL UNITARITY}

Before we explain how to find numerical solutions to the nonlinear integral equations (14) in the presence of anomalous threshold effects it is useful to pause and discuss a critical issue that we will be confronted with. Given a specific approximation for the generalized potential, a solution of Eq. (14) does not necessarily imply that the coupled-channel unitarity condition is fulfilled. From the latter we expect a representation of the reaction amplitudes $T_{a b}(s)$ in terms of a set of real quantities, i.e., the channel-dependent phase shift and inelasticity parameters $\phi_{a}(s)$ and $\eta_{a}(s)$. It should hold that

$$
\begin{aligned}
\operatorname{Im} T_{a b}(s+i \epsilon)= & \sum_{c, d} T_{c a}^{*}(s+i \epsilon) \rho_{c d}(s) T_{d b}(s+i \epsilon) \\
& \times \Theta\left[s-\left(m_{c}+M_{c}\right)^{2}\right] \\
& \text { for } s>\operatorname{Max}\left\{\left(m_{a}+M_{a}\right)^{2},\left(m_{b}+M_{b}\right)^{2}\right\}, \\
\sum_{c} T_{a c}(s) \rho_{c a}(s)= & \frac{1}{2 i}\left[\eta_{a}(s) e^{2 i \phi_{a}(s)}-1\right],
\end{aligned}
$$

where on general grounds one expects $0 \leq \eta_{a} \leq 1$. While any solution to Eq. (14) satisfies the time-reversal invariance condition $T_{a b}(s)=T_{b a}(s)$, the Schwarz reflection principle, 


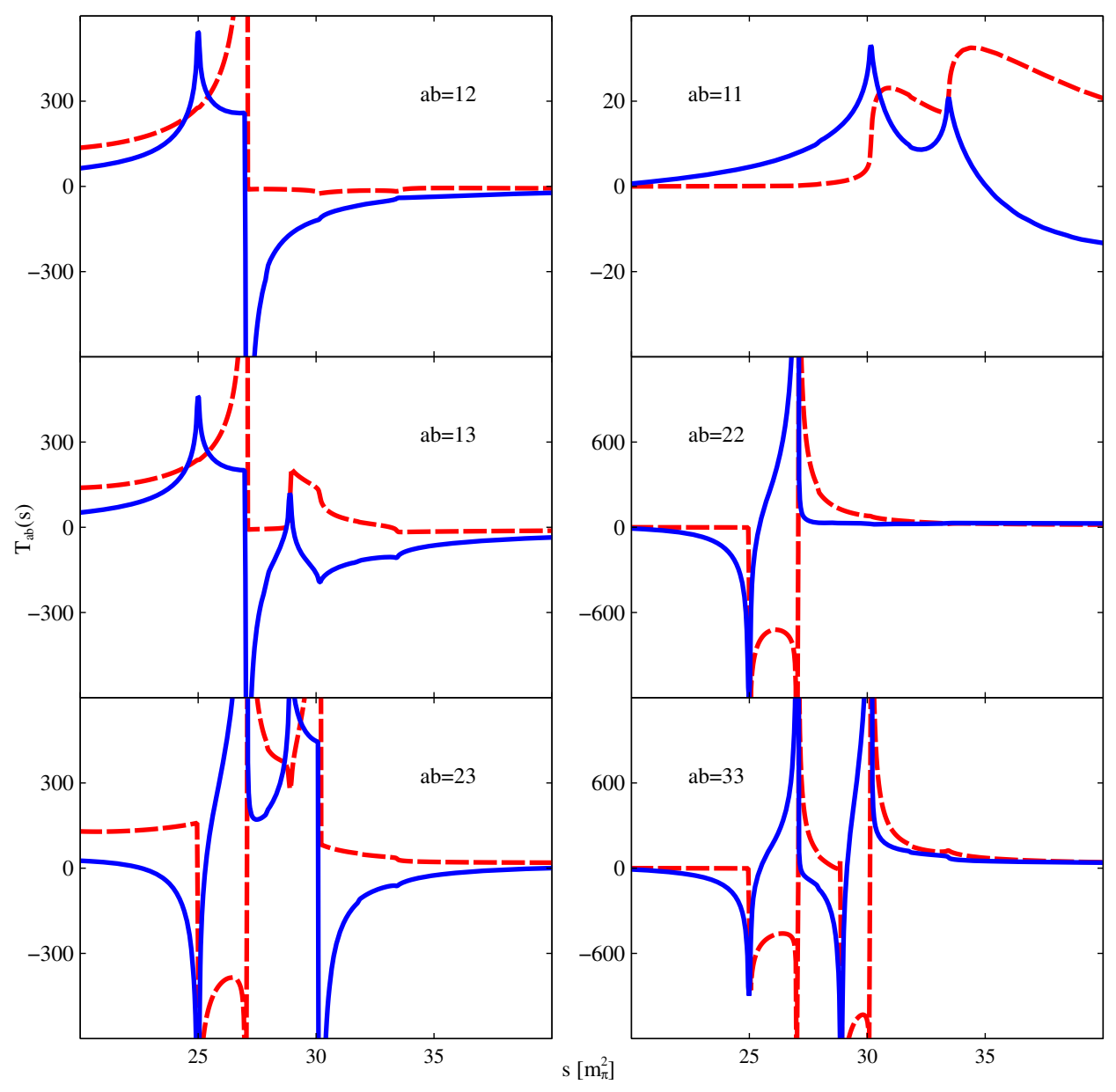

FIG. 5. Reaction amplitudes $T_{a b}(s+i \epsilon)=T_{b a}(s+i \epsilon)$ in the schematic model of Eq. (15). Real and imaginary parts are shown with solid blue and dashed red lines respectively.

$$
T_{a b}^{*}(s)=T_{a b}\left(s^{*}\right),
$$

cannot be derived in general for right-hand cut lines off the real axis. It is not surprising then that the coupled-channel unitarity condition is not necessarily obtained.

Let us be specific and identify the generalized potential by tree-level $t$ - and $u$-channel exchange processes, a typical strategy in hadron physics. Though we may solve the nonlinear system (14) in this case, the unitarity condition (14) will not be fulfilled once an anomalous threshold effect is encountered. A supposedly related problem was noted in the previous studies $[37,38]$. It was argued that the problem is caused by the neglect of second-order contributions to the generalized potential. A minimal ansatz for a physical approximation to the generalized potential requires some additional terms

$$
U_{a b}(s)=U_{a b}^{\mathrm{tree}-\mathrm{level}}(s)+U_{a b}^{\mathrm{box}}(s),
$$

to be constructed properly. However, no conclusive form of the latter has been presented and illustrated in the literature so far. Conflicting suggestions were put forward in Refs. [37,38] for two-channel systems. Whether such forms lead to physical results remains an open challenge. So far there is no numerical implementation for a specific example worked out, at hand of which one may judge the significance of any of the two approaches. Unfortunately, for us both works $[37,38]$ are rather difficult to follow. Nevertheless, we tend to agree with the conclusions of Ref. [38] that the approach advocated in Ref. [37] is incorrect.

An important achievement of the current work is the construction of a minimal second-order term applicable for systems of arbitrarily high dimensions. Our approach is based on the request that we arrive at the coupled-channel unitarity condition (16) and recover the Schwarz reflection principle (17). The key observation is that in the absence of the anomalous box term $U_{a b}^{\text {box }}(s)$ the second-order reaction amplitude $T_{a b}(s)$, as it would follow from Eq. (14), is at odds with Eq. (17). The extra term is unambiguously determined by the condition that its inclusion restores the Schwarz reflection principle (17). This leads to the following form: 


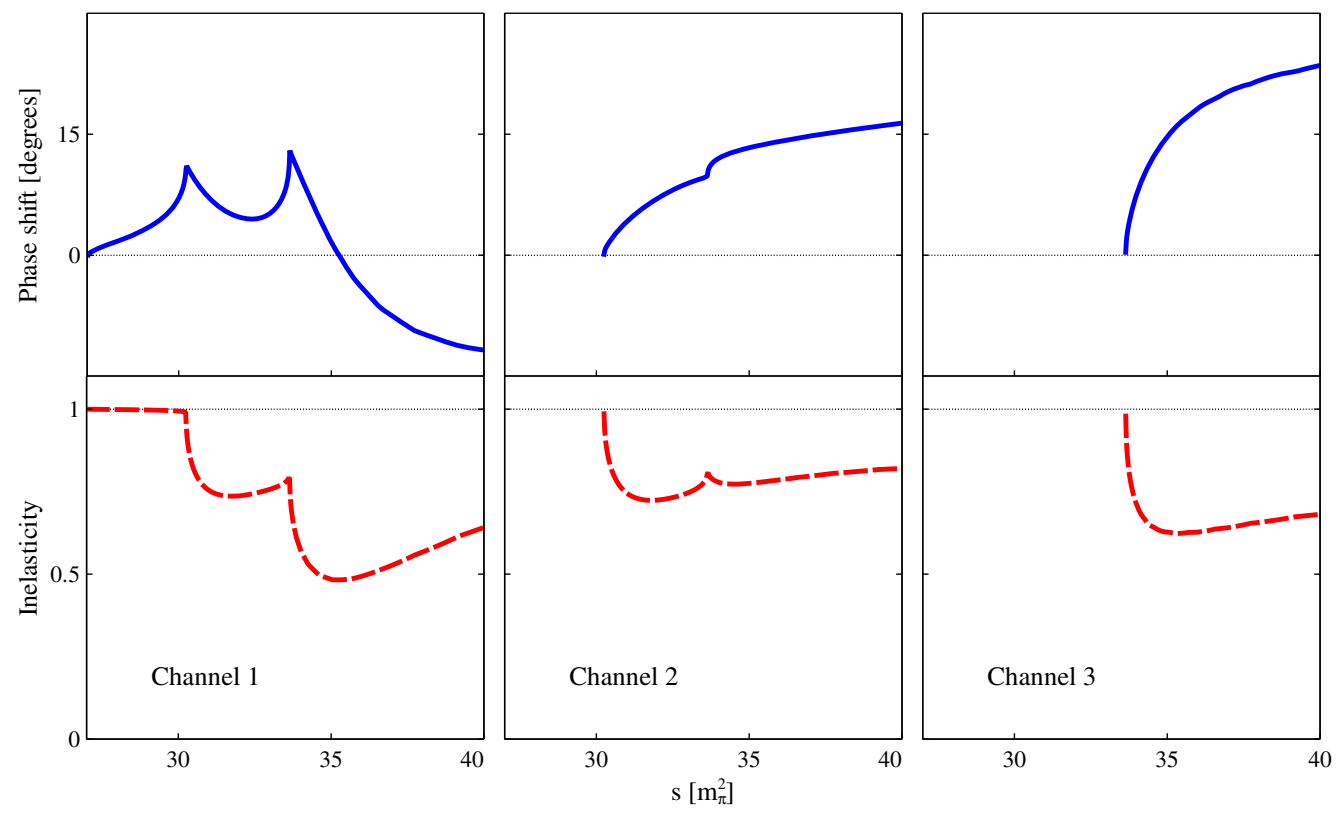

FIG. 6. Phase shifts and inelasticity parameters of Eq. (16) in our schematic model (15).

$$
\begin{aligned}
U_{a b}^{\text {tree-level }}(s) & =\int_{\Delta L_{a b}} \frac{d \bar{s}}{\pi} \frac{\rho_{a b}^{\text {tree-level }}(\bar{s})}{\bar{s}-s}+\int_{A_{\operatorname{Min}(a, b)}} \frac{d \bar{s}}{\pi} \frac{\rho_{a b}^{A}(\bar{s})}{\bar{s}-s}, \\
U_{a b}^{\mathrm{box}}(s) & =2 \sum_{c, d<\operatorname{Min}(a, b)} \int_{A_{c}} \frac{d \bar{s} \rho_{a c}^{A}(\bar{s}) \rho_{c d}(\bar{s}) \rho_{c b}^{A}(\bar{s})}{\bar{s}-s},
\end{aligned}
$$

where the spectral weight $\rho_{a b}^{A}(\bar{s})$ characterizes the anomalous behavior of the tree-level potential. It is identified in analogy to the general representation (12). Unfortunately, a direct comparison of our result with the ansatz in Refs. [37,38] is not so easy. Nevertheless, we note that for the two-channel case our result (19) appears quite compatible with the ansatz discussed in Ref. [38].

We anticipate the phase shifts and inelasticities as implied by our schematic model (15) as properly supplemented by the anomalous box term (18)-(19). We affirm that the reaction amplitudes as already shown in Fig. 5 are compatible with the coupled-channel unitarity condition (16) and the phase shifts and inelasticity parameters of Fig. 6. Without an explicit computation the authors would not have been in a position to even roughly guess the nontrivial behavior seen in that figure.

It is instrumental to realize the quite different nature of the two contributions in Eq. (18). Consider first the tree-level term in Eqs. (18)-(19). The spectral weight $\rho_{a b}^{A}(s)$ is real as $s$ approaches the real line below $s<\operatorname{Min}\left\{\left(m_{a}+M_{a}\right)^{2}\right.$, $\left.\left(m_{b}+M_{b}\right)^{2}\right\}$. In contrast it is purely imaginary for $s>\operatorname{Min}\left\{\left(m_{a}+M_{a}\right)^{2},\left(m_{b}+M_{b}\right)^{2}\right\}$. This follows from the general results presented in Ref. [28]. The corresponding generalized potential satisfies the Schwarz reflection principle with

$$
\left[U_{a b}^{\text {tree-level }}(s)\right]^{*}=U_{a b}^{\text {tree-level }}\left(s^{*}\right) .
$$

We turn to the second-order term in Eqs. (18)-(19). While for $\operatorname{Re} s<\left(m_{c}+M_{c}\right)^{2}=\left(m_{d}+M_{d}\right)^{2}$ the two factors, $\rho_{a c}^{A}(s)$ and $\rho_{c b}^{A}(s)$, are real quantities as $s$ approaches the real line, the phase-space factor $\rho_{c d}(s)$ turns purely imaginary in this case. This leads to the property

$$
\left[U_{a b}^{\mathrm{box}}(s)\right]^{*}=-U_{a b}^{\mathrm{box}}\left(s^{*}\right),
$$

and illustrates the particular feature of the anomalous box term our construction is based on. Given our schematic model (15) the anomalous box term $U_{a b}^{\text {box }}(s)$ is illustrated with Fig. 7. Like in Fig. 6 the left-hand panels show the nonvanishing potentials on the real axis, while the righthand panels show the corresponding potentials slightly below the real axis at $s-i \epsilon$.

It should be emphasized that there are further secondorder contributions to the generalized potential; however, they add to the "normal" spectral weight $\rho_{a b}^{\text {normal }}(\bar{s})$ in Eq. (12) only. Since the latter terms do not jeopardize the coupled-channel unitarity condition, there is no stringent reason to consider such effects in an initial computation. Typically one may hope that the effect of the latter is suppressed in some suitable power-counting scheme. This should be so since higher-loop effects are characterized by left-hand branch cuts that are further separated from the right-hand cuts. In turn such contributions to the generalized potential cannot show any significant variations at energies where the generalized potential is needed in Eq. (14).

We would speculate, that the ansatz (18) is quite generic, i.e., it should hold also for contributions including higherloop effects 


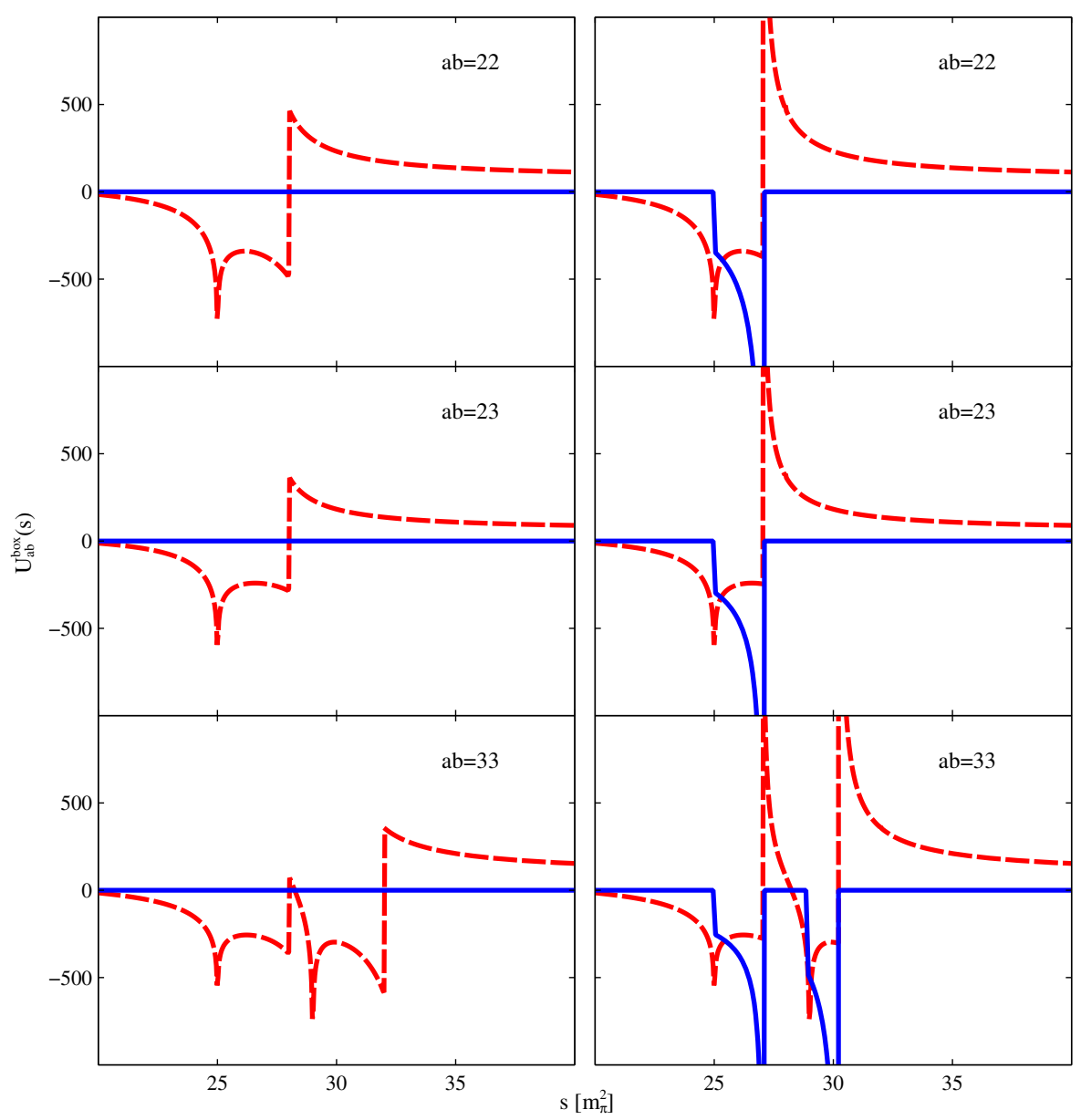

FIG. 7. The box contributions $U_{a b}^{\text {box }}(s)$ (left panels) and $U_{a b}^{\text {box }}(s-i \epsilon)$ (right panels) as defined in Eq. (19) with our model input (15). Only nonvanishing elements are considered. Real and imaginary parts are shown with solid blue and dashed red lines respectively.

$$
\begin{aligned}
U_{a b}(s)= & \int_{\Delta L_{a b}} \frac{d \bar{s}}{\pi} \frac{\rho_{a b}^{\text {normal }}(\bar{s})}{\bar{s}-s}+\int_{A_{\operatorname{Min}(a, b)} \frac{d \bar{s}}{\pi} \frac{\rho_{a b}^{\text {anomalous }}(\bar{s})}{\bar{s}-s}} \\
& +\sum_{c<\operatorname{Min}(a, b)} \int_{A_{c}} \frac{d \bar{s}}{\pi} \frac{\rho_{a b, c}^{\text {anomalous }}(\bar{s})}{\bar{s}-s}
\end{aligned}
$$

where we expect $\rho_{a b, c}^{\text {anomalous }}(\bar{s})$ to be determined by $\rho_{a c}^{\text {anomalous }}(\bar{s})$ and $\rho_{c b}^{\text {anomalous }}(\bar{s})$ in analogy to Eq. (19).

\section{NONLINEAR INTEGRAL EQUATION ON COMPLEX CONTOURS}

The key issue is how to numerically solve the nonlinear set of equations (14) and cross-check its physical correctness. After all one may consider it merely as a definition of the generalized potential in the presence of anomalous thresholds. For a given approximated generalized potential $U_{a b}(s)$ we will devise an appropriate $N / D$-like ansatz that will eventually lead to a framework which is amenable to numerical simulations of Eq. (14).
We introduce a set of contour functions $\varsigma_{a b}(\bar{s})$ defined initially on distinct contours $C_{b}$, the choice of which depends on the channel index $b$

$$
\begin{aligned}
\varsigma_{a b}(\bar{s}) & =-\sum_{c, d} D_{a c}^{b+}(\bar{s}) T_{c d}^{b+}(\bar{s}) \rho_{d b}(\bar{s}) \\
& =-\sum_{c, d} D_{a c}^{b-}(\bar{s}) T_{c d}^{b-}(\bar{s}) \rho_{d b}(\bar{s}) \quad \text { for } \bar{s} \in C_{b}, \\
D_{a b}(s) & =\delta_{a b}+\int_{C_{b}} \frac{d \bar{s}}{\pi} \frac{s-\mu^{2}}{\bar{s}-\mu^{2}} \frac{\varsigma_{a b}(\bar{s})}{\bar{s}-s},
\end{aligned}
$$

where we apply the convenient \pm notation introduced already in Eq. (13). Assuming the existence of such a set of functions $\varsigma_{a b}(\bar{s})$ we seek to express the reaction amplitude $T_{a b}(s)$ in terms of them. This requires a few steps. Like in the previous sections we anticipate with Fig. 8 the form of the $D$ functions as they are implied in our schematic model. This may help the reader to fight through the various abstract arguments presented in the following. It is emphasized that none of the functions $D_{a b}(s+i \epsilon)$ depend on the particular choice of the return points $\hat{\mu}_{a b}$ in Eq. (15). The 

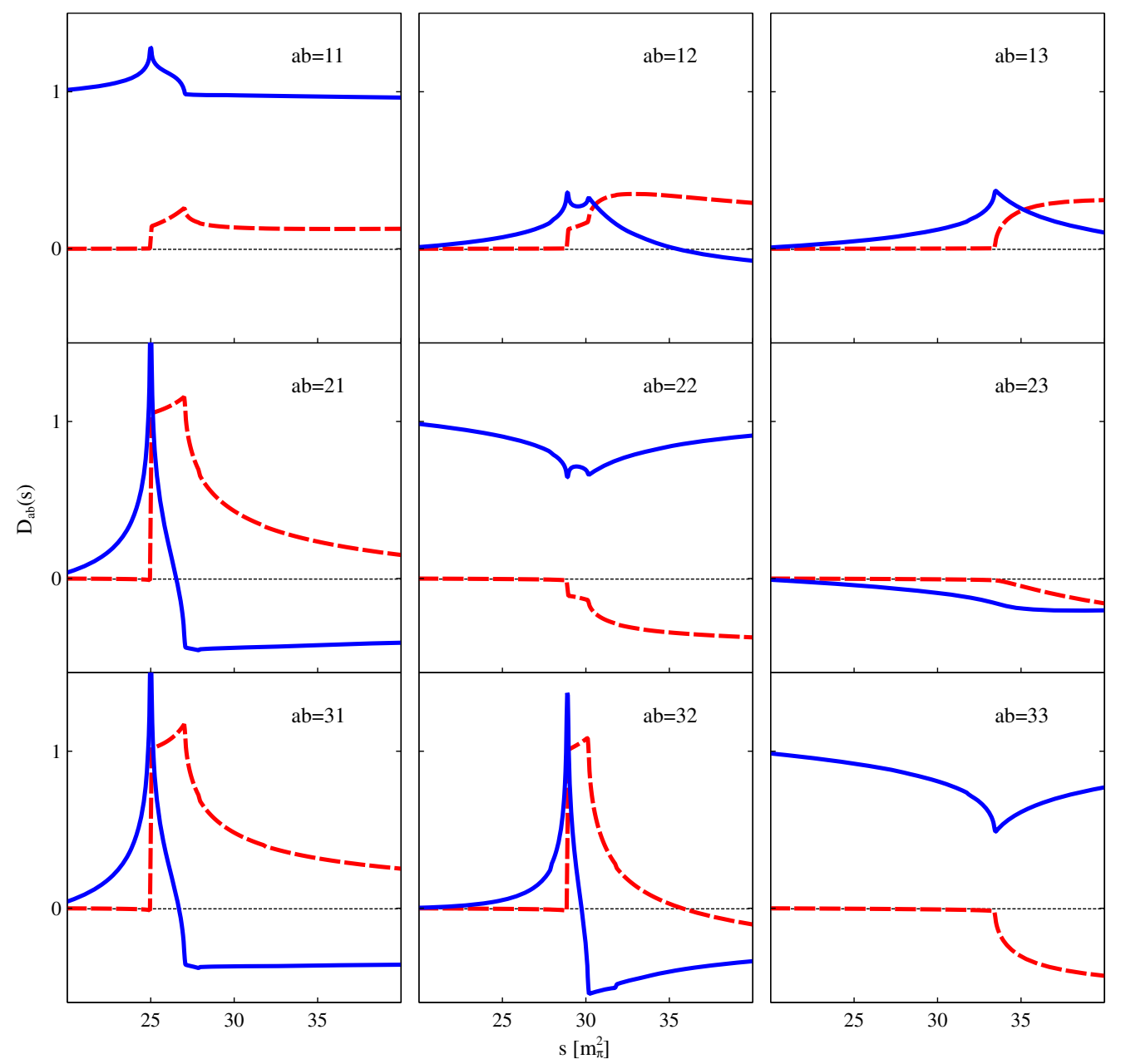

FIG. 8. The functions $D_{a b}(s+i \epsilon)$ of Eq. (23) in our schematic model (15). Real and imaginary parts are shown with solid blue and dashed red lines respectively.

functions have a significant imaginary part starting at the anomalous threshold $s \geq \mu_{b}^{2}$.

In the first step we study the analytic properties of the function

$$
\begin{aligned}
B_{a b}(s) & \equiv \sum_{d} D_{a d}(s)\left[U_{d b}(s)-T_{d b}(s)\right] \\
& =-\sum_{c, d, e} D_{a d}(s) \int_{C_{c}} \frac{d \bar{s}}{\pi} \frac{s-\mu^{2}}{\bar{s}-\mu^{2}} \frac{T_{d c}^{c-}(\bar{s}) \rho_{c e}(\bar{s}) T_{e b}^{c+}(\bar{s})}{\bar{s}-s},
\end{aligned}
$$

where we applied the master equation (14). In Fig. 9 we provide such functions $B_{a b}(s+i \epsilon)$ as implied by our model (15) for $s>\left(m_{b}+M_{b}\right)^{2}$, a region which encompasses the physical domain probed by the phase shifts and inelasticity parameters in Eq. (16). Here we again do not encounter any dependence on the return points $\hat{\mu}_{a}$. However, the functions $B_{a 1}(s)$ show a strong variation close to the anomalous threshold point at $s=\mu_{2}^{2}=29 m_{\pi}^{2}$. It is important to note that this is not propagated into the amplitude $T_{11}(s+i \epsilon)$ as is evident from Fig. 5 and Fig. 6.
From Eq. (23) it follows that the function $D_{a b}(s)$ is analytic in the complex plane with the exception of a cut along the contour $C_{b}$. A similar conclusion can be drawn for the function $B_{a b}(s)$ only that in this case the cut line is a superposition of all right-hand contours $C_{c}$. Since the contours $C_{c}$ partially overlap it is useful to decompose the contours with

$C_{c}=C_{c}^{+}+C_{c}^{-}$and $C_{A}=C_{1}^{-} \rightarrow \sum_{c} C_{c}=C_{A}+\sum_{c} C_{c}^{+}$,

where the lower $C_{c}^{-}$contours are all on the straight line crossing the value $s=-i \epsilon$. By construction the upper contours $C_{c}^{+}$do not overlap.

From this we conclude that a dispersion integral representation of the form

$$
\begin{aligned}
B_{a b}(s)= & \frac{1}{2 i} \sum_{c} \int_{C_{c}^{+}} \frac{d \bar{s} s-\mu^{2}}{\pi} \frac{B_{a b}^{c+}(\bar{s})-B_{a b}^{c-}(\bar{s})}{\bar{s}-s} \\
& +\frac{1}{2 i} \int_{C_{A}} \frac{d \bar{s}}{\pi} \frac{s-\mu^{2}}{\bar{s}-\mu^{2}} \frac{B_{a b}^{A+}(\bar{s})-B_{a b}^{A-}(\bar{s})}{\bar{s}-s},
\end{aligned}
$$




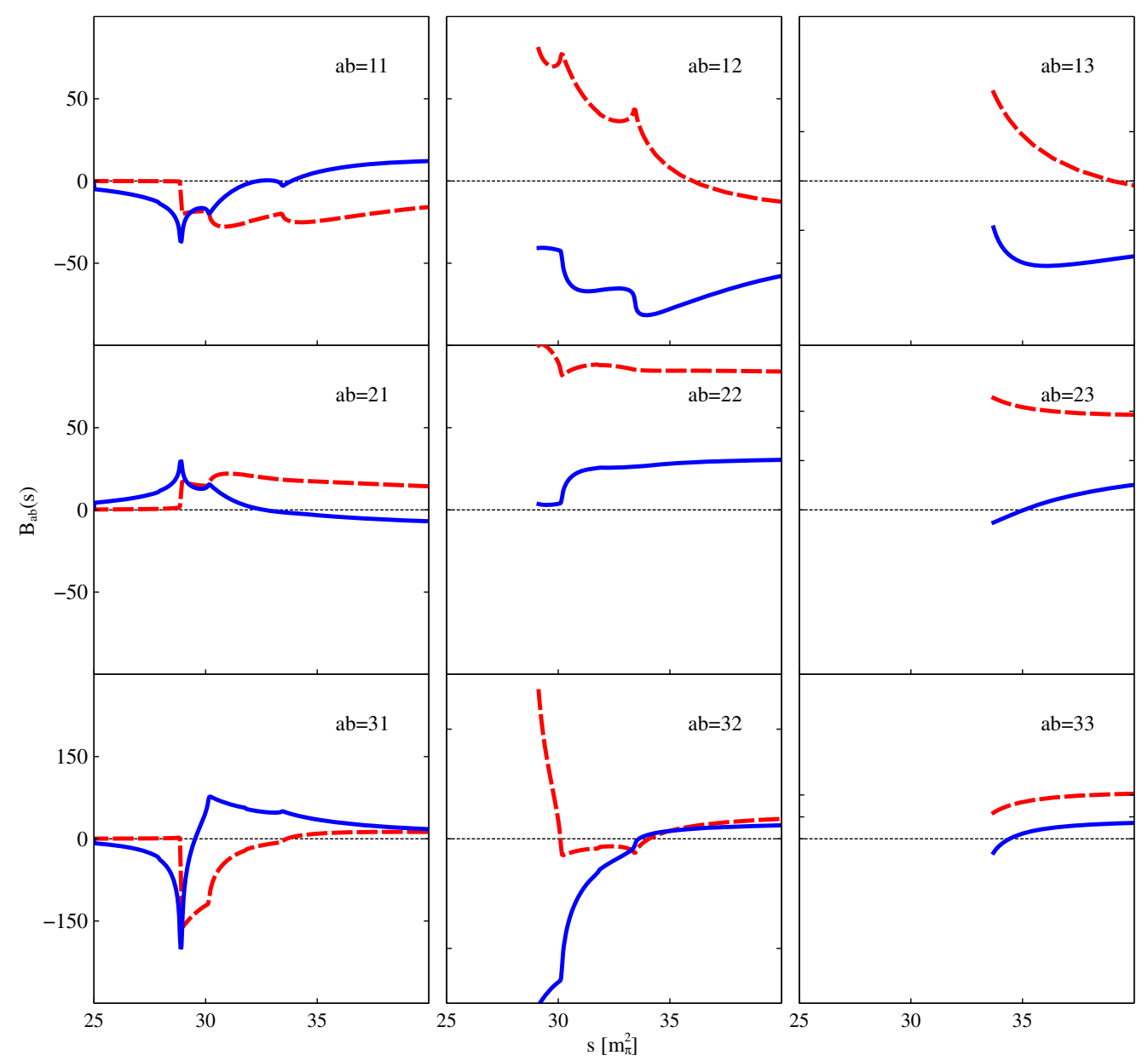

FIG. 9. The functions $B_{a b}(s+i \epsilon)$ of Eq. (24) in our schematic model (15) for $s>\mu_{b}^{2}$ or $s>\left(m_{b}+M_{b}\right)^{2}$. Real and imaginary parts are shown with solid blue and dashed red lines respectively.

can be assumed. In the next step we compute the discontinuity along the contours $C_{c}^{+}$with

$$
\begin{aligned}
\frac{1}{2 i}\left[B_{a b}^{c+}(\bar{s})-B_{a b}^{c-}(\bar{s})\right] & =\frac{1}{2} \varsigma_{a c}(\bar{s})\left[U_{c b}^{c+}(\bar{s})+U_{c b}^{c-}(\bar{s})\right]-\frac{1}{2} \varsigma_{a c}(\bar{s})\left[T_{c b}^{c+}(\bar{s})+T_{c b}^{c-}(\bar{s})\right]-\frac{1}{2} \sum_{d}\left[D_{a d}^{c+}(\bar{s})+D_{a d}^{c-}(\bar{s})\right] \sum_{e} T_{d c}^{c-}(\bar{s}) \rho_{c e}(\bar{s}) T_{e b}^{c+}(\bar{s}) \\
& =\frac{1}{2} \varsigma_{a c}(\bar{s})\left[U_{c b}^{c+}(\bar{s})+U_{c b}^{c-}(\bar{s})\right],
\end{aligned}
$$

where we observe the cancellation of most terms in Eq. (27). This follows from the defining equations for $\varsigma_{a b}(\bar{s})$ in Eq. (23) together with the symmetry of the reaction amplitude

$$
\begin{aligned}
U_{a b}(s) & =U_{b a}(s) \quad \& \quad \rho_{a b}(s)=\rho_{b a}(s), \\
\rightarrow T_{a b}(s) & =T_{b a}(s) \quad \& \quad \sum_{e} T_{d c}^{c-}(\bar{s}) \rho_{c e}(\bar{s}) T_{e b}^{c+}(\bar{s})=\sum_{e} T_{d c}^{c+}(\bar{s}) \rho_{c e}(\bar{s}) T_{e b}^{c-}(\bar{s}) .
\end{aligned}
$$

It is left to compute the discontinuity of the $B$ functions along the straight contour $C_{A}$, i.e., the second term in Eq. (26) is considered. For any $\bar{s} \in C_{A}$ we derive

$$
\begin{aligned}
\frac{1}{2 i}\left[B_{a b}^{A+}(\bar{s})-B_{a b}^{A-}(\bar{s})\right]= & \frac{1}{2} \sum_{c} \varsigma_{a c}(\bar{s})\left[U_{c b}^{c+}(\bar{s})+U_{c b}^{c-}(\bar{s})\right] \Theta\left[\bar{s}+i \epsilon-\mu_{c}^{2}\right]-\frac{1}{2} \sum_{c} \varsigma_{a c}(\bar{s})\left[T_{c b}^{c+}(\bar{s})+T_{c b}^{c-}(\bar{s})\right] \Theta\left[\bar{s}+i \epsilon-\mu_{c}^{2}\right] \\
& -\frac{1}{2} \sum_{c, d}\left[D_{a d}^{c+}(\bar{s})+D_{a d}^{c-}(\bar{s})\right] \sum_{e} T_{d c}^{c-}(\bar{s}) \rho_{c e}(\bar{s}) T_{e b}^{c+}(\bar{s}) \Theta\left[\bar{s}+i \epsilon-\mu_{c}^{2}\right] \\
& =\frac{1}{2} \sum_{c} \varsigma_{a c}(\bar{s})\left[U_{c b}^{c+}(\bar{s})+U_{c b}^{c-}(\bar{s})\right] \Theta\left[\bar{s}+i \epsilon-\mu_{c}^{2}\right]
\end{aligned}
$$


where the cancellations in Eq. (29) follow from Eqs. (23) and (28). We observe that owing to the identities (27) and (29) the two terms in Eq. (26) can be combined into a dispersion integral written in terms of the partially overlapping contours $C_{c}$. It holds that

$$
B_{a b}(s)=\sum_{c} \int_{C_{c}} \frac{d \bar{s}}{\pi} \frac{s-\mu^{2}}{\bar{s}-\mu^{2}} \frac{\varsigma_{a c}(\bar{s})}{\bar{s}-s} U_{c b}(\bar{s}),
$$

with $U_{a b}(s)=U_{a b}^{c+}(s)=U_{a b}^{c-}(s)$ for

$$
s \in C_{c} \text { given any } c,
$$

where the crucial identity in the last line of Eq. (30) is a consequence of the properly deformed contour lines as illustrated in Fig. 3.

With this we arrive at the anticipated representation of the scattering amplitude in terms of the spectral density $\varsigma_{a b}(\bar{s})$. It holds that

$$
\begin{aligned}
T_{a b}(s) & =U_{a b}(s)-\sum_{c} D_{a c}^{-1}(s) B_{c b}(s) \\
& =U_{a b}(s)-\sum_{c, d} D_{a d}^{-1}(s) \int_{C_{c}} \frac{d \bar{s}}{\pi} \frac{s-\mu^{2}}{\bar{s}-\mu^{2}} \frac{s_{d c}(\bar{s})}{\bar{s}-s} U_{c b}(\bar{s}) .
\end{aligned}
$$

The functions $D_{a b}(s)$ were already expressed in terms of $\varsigma_{a b}(\bar{s})$ in Eq. (23).

We are one step away from a more practical form of the defining requirement (23) and in particular checking the consistency of the construction. After all we had to use both equations in the first line of Eq. (23) to arrive at Eq. (31). By inserting our result (31) into Eq. (23) we obtain two distinct equations, which we must show to be equivalent. We derive

$$
\begin{aligned}
\varsigma_{a b}(s)= & -\sum_{d} U_{a d}(s) \rho_{d b}(s) \\
& -\sum_{c, d} \int_{C_{c}} \frac{d \bar{s} s-\mu^{2}}{\pi} \frac{\varsigma_{a c}(\bar{s})}{\bar{s}-\mu^{2}}\left[U_{c d}(s)-U_{c d}(\bar{s})\right] \rho_{d b}(s),
\end{aligned}
$$

where $s$ is strictly on the contour $C_{b}$. With $s_{ \pm}$we introduce values of $s$ slightly above and below the contour $C_{b}$, i.e., it holds that $\left|s-s_{ \pm}\right|<\epsilon^{\prime}$, where $\epsilon^{\prime}$ is chosen smaller than the minimal distance of any of the horizontal lines in Fig. 3. The two choices correspond to the two identities in the first line of Eq. (23). Since the numerator in Eq. (32) strictly vanishes at $\bar{s}=s$ both choices lead to identical results.

While with Eq. (32) we arrive at a mathematically welldefined linear integral equation, it remains to construct a numerical solution to it. This is not quite straightforward and will require further developments. In the following we will analyze the linear system (32) in more detail and eventually establish a framework that can be used to numerically solve it on a computer. The key issue is to systematically perform the limit $\epsilon \rightarrow 0$ in the system of complex contours.

\section{FROM COMPLEX CONTOURS TO REAL CONTOURS}

We consider first the $D$ function, whose definition is

$$
D_{a b}(s)=\delta_{a b}+\int_{C_{b}} \frac{d \bar{s}}{\pi} \frac{s-\mu^{2}}{\bar{s}-\mu^{2}} \frac{\varsigma_{a b}(\bar{s})}{\bar{s}-s},
$$

in terms of the spectral weight $\varsigma_{a b}(s)$. We consider the limit $\epsilon \rightarrow 0$, in which the complex contours $C_{c}$ all approach the real axis. If we are only interested in values of $s$ that are below or above all right-hand cut lines, we may simplify the integral into Riemann sums on the real axis. This is achieved as follows.

We first note that we may consider $\varsigma_{a c}(s)$ to be an analytic function in $s$, with various branch cuts. This follows from the integral representation (32). More precisely, if the linear system has a solution $\varsigma_{a c}(s)$ with $s \in C_{c}$ then Eq. (32) can be used to analytically continue $\varsigma_{a c}(s)$ away from the contour line $C_{c}$. The branch cuts are readily identified. First it carries the branch cuts of the phase-space function $\rho_{c c}(s)$ that is strictly on the real axis in our convention. Second, the cut lines of the generalized potential $U_{a c}(s)$ for any $a$ are inherited. The important observation is the absence of any right-hand cut lines.

According to the cut lines summarized in Fig. 3 there are two critical points, $\mu_{b}$ and $\hat{\mu}_{b}$, associated with a normal threshold point at $m_{b}+M_{b}$. While $\mu_{b}$ denotes the smallest anomalous threshold opening of the generalized potential $U_{a b}(s)$ with arbitrary $a$, the return point $\hat{\mu}_{b}$, specifies the point at which the left-hand contour line circles around the point $s=\left(m_{b}+M_{b}\right)^{2}$ in Fig. 3 and comes back. With this in mind we introduce

$$
\begin{aligned}
D_{a b}(s)= & \delta_{a b}+\int_{\left(m_{b}+M_{b}\right)^{2}}^{\infty} \frac{d \bar{s}}{\pi} \frac{s-\mu^{2}}{\bar{s}-\mu^{2}} \frac{\hat{s}_{a b}(\bar{s})}{\bar{s}-s} \\
& +\int_{\mu_{b}^{2}}^{\hat{\mu}_{b}^{2}} \frac{d \bar{s}}{\pi} \frac{s-\mu^{2}}{\bar{s}-\mu^{2}} \frac{\Delta \varsigma_{a b}(\bar{s})}{\bar{s}-s},
\end{aligned}
$$

with $\hat{\varsigma}_{a b}(\bar{s})=\varsigma_{a b}^{+}(\bar{s}) \Theta\left(\hat{\mu}_{b}^{2}-\bar{s}\right)+\varsigma_{a b}^{-}(\bar{s}) \Theta\left(\bar{s}-\hat{\mu}_{b}^{2}\right)$,

$$
\Delta \varsigma_{a b}(\bar{s})=\varsigma_{a b}^{-}(\bar{s})-\varsigma_{a b}^{+}(\bar{s}),
$$

where the integrals over $\bar{s}$ in Eq. (34) are strictly on the real axis. In Eq. (34) we apply the useful notation

$$
\begin{aligned}
& \varsigma_{a b}^{ \pm}(\bar{s})=\varsigma_{a b}\left(s_{ \pm}\right) \quad \text { with } \operatorname{Im} \bar{s}=0 \text { and } s_{ \pm} \in C_{b} \\
& \text { and } \operatorname{Im}\left(s_{+}-s_{-}\right)>0 \text { and } \operatorname{Re} s_{ \pm}=\bar{s},
\end{aligned}
$$

which initially only defines $\varsigma_{a b}^{+}(\bar{s})$ for $\bar{s}<\left(m_{b}+M_{b}\right)^{2}$, but is naturally extended up to the point $\bar{s}=\hat{\mu}_{b}^{2}$. Here we assume the availability of the analytic continuation of the function $\varsigma_{a b}(s)$ from the nominal threshold value at 


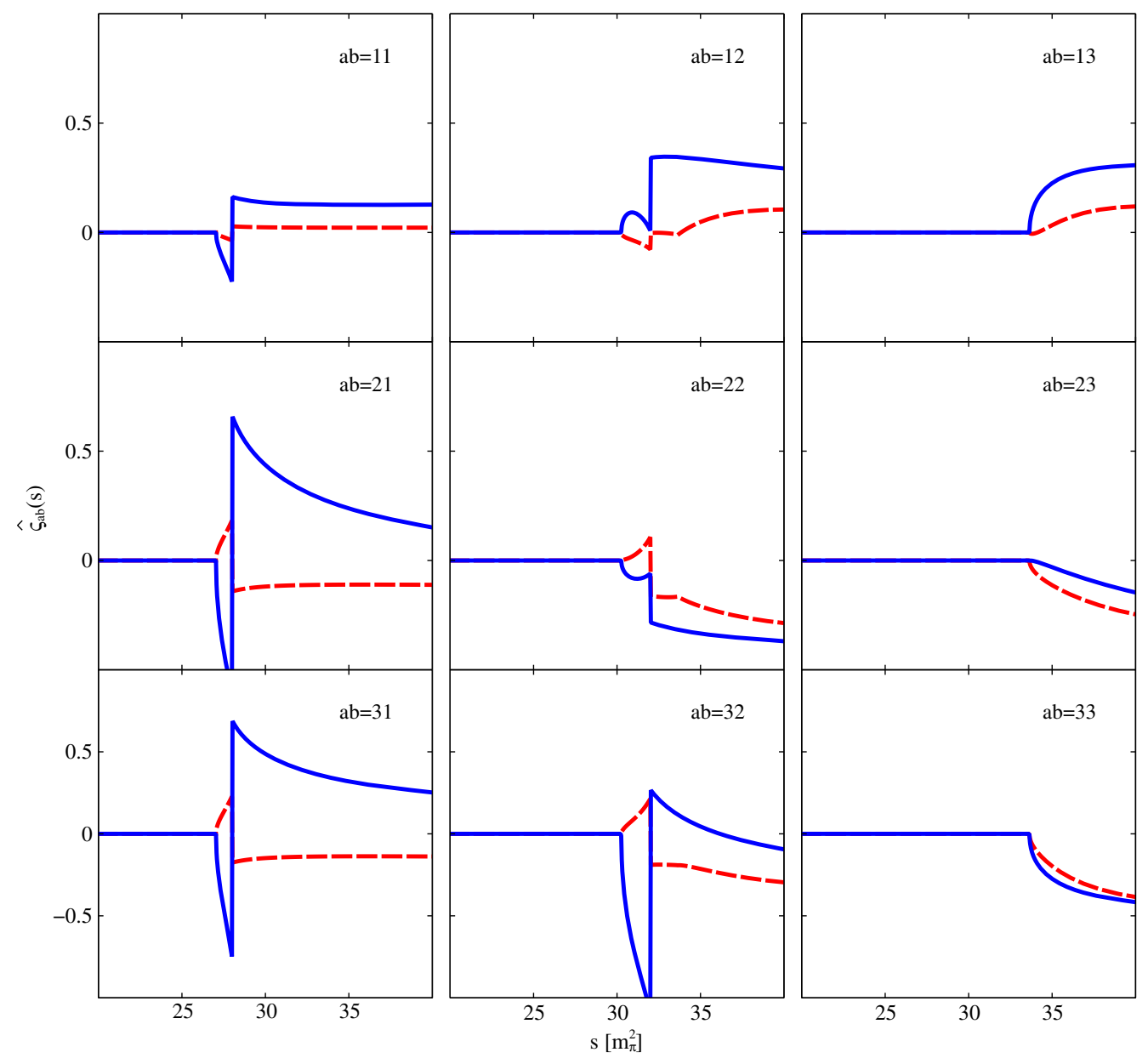

FIG. 10. The function $\hat{\varsigma}_{a b}(s)$ of Eqs. (34) and (54) in our schematic model (15). Real and imaginary parts are shown with solid blue and dashed red lines respectively.

$s=\left(m_{b}+M_{b}\right)^{2}$ up to the return point of the left-hand cut at $s=\hat{\mu}_{b}^{2}$ in Fig. 3. The particular location of $\hat{\mu}_{b}>m_{b}+$ $M_{b}$ is irrelevant. While the spectral weights $\hat{\varsigma}_{a b}(\bar{s})$ and $\Delta \varsigma_{a b}(\bar{s})$ depend on it, by construction the $D$ function does not. Given Eq. (35) the limit $\epsilon \rightarrow 0$ in the right- and lefthand contours of Fig. 3 can be applied without changing the form of Eq. (34). In this limit the contributions of any vertical parts of the contour lines vanish. Such terms are already omitted altogether in Eq. (34). Note that modulo those vertical lines Eq. (34) is nothing but a regrouping of the various contour contributions in Eq. (33).
We illustrate the generic form of the spectral weight $\hat{\varsigma}_{a b}(s)$ in our model (15). As shown in Fig. 10 the complex functions are nonzero for $s>\left(m_{b}+M_{b}\right)^{2}$ only. It is important to note that the latter do depend on the choice of the return point $\hat{\mu}_{b}$. The functions $\hat{\varsigma}_{a b}(s)$ are piecewise continuous with the only discontinuous behavior being at the return point $s=\hat{\mu}_{b}^{2}$.

The result (34) is useful since in the limit $\epsilon \rightarrow 0$ the anomalous spectral weight $\Delta \varsigma_{a c}(\bar{s})$ can be linked back to the $D$ function as follows. A direct application of Eq. (32) leads to

$$
\begin{aligned}
\Delta \varsigma_{a b}(\bar{s}) & =\sum_{c>b, d}\left[D_{a c}\left(\bar{s}_{+}\right) U_{c d}\left(\bar{s}_{+}\right)-D_{a c}\left(\bar{s}_{-}-i \epsilon^{\prime}\right) U_{c d}\left(\bar{s}_{-}\right)\right] \Theta\left[\left(\mu_{c d}^{A}\right)^{2}<\bar{s}<\hat{\mu}_{b}^{2}\right] \rho_{d b}(\bar{s}) \\
& =-\sum_{c>b, d} D_{a c}\left(\bar{s}+i \epsilon^{\prime}\right)\left[U_{c d}^{-d}(\bar{s})-U_{c d}^{+d}(\bar{s})\right] \Theta\left[\left(\mu_{c d}^{A}\right)^{2}<\bar{s}<\hat{\mu}_{b}^{2}\right] \rho_{d b}(\bar{s})+2 i \sum_{c>b, d} \Delta \varsigma_{a c}(\bar{s}) U_{c d}^{-d}(\bar{s}) \Theta\left[\mu_{c}^{2}<\bar{s}<\hat{\mu}_{b}^{2}\right] \rho_{d b}(\bar{s}), \\
U_{c d}^{ \pm b}(\bar{s}) & =U_{c d}\left(\bar{s}_{ \pm}\right) \quad \text { with } \quad \operatorname{Im} \bar{s}=0 \quad \text { and } \quad \bar{s}_{ \pm} \in C_{b} \quad \text { and } \quad \operatorname{Im}\left(\bar{s}_{+}-\bar{s}_{-}\right)>0 \quad \text { and } \quad \operatorname{Re} \bar{s}_{ \pm}=\bar{s}
\end{aligned}
$$

where we used the crucial property that the generalized potential $U_{a b}(s)$ may develop an anomalous threshold behavior at the lower of the two nominal thresholds at $s=\left(m_{a}+M_{a}\right)^{2}$ or $s=\left(m_{b}+M_{b}\right)^{2}$. Given our strict channel ordering the sum in Eq. (36) over the channel index $c$ is restricted to the case $c>b$. 
There is a subtle point as to where to evaluate the $D_{a c}\left(\bar{s}_{-}-i \epsilon^{\prime}\right)$ function in the first line of Eq. (36). Since $D_{a c}(s)$ has a branch cut along $C_{c}$ and $\bar{s}_{-} \in C_{c}$ for $\bar{s}>\mu_{c}^{2}$ it is necessary to specify whether we should evaluate the function below or above the cut. Our prescription follows unambiguously if we slightly deform the contours $C_{c}$ in Eq. (32). In Fig. 3 the solid line passing through $\bar{s}_{-}$is deformed a bit towards, but still avoids, the dashed lines above. With this it is manifest from Eq. (32) that $\varsigma_{a b}(s)$ is analytic along the horizontal line through $\bar{s}_{-}$. This should be so since $\varsigma_{a b}(s)$ is analytic along all contours $C_{c}$ by construction. In turn our prescription $D_{a c}\left(\bar{s}_{-}-i \epsilon^{\prime}\right)$ is justified. Note that for the term $D_{a c}\left(\bar{s}_{+}\right)$no further specification is needed simply because $\bar{s}_{+} \notin C_{c}$. Here it always holds that $D_{a c}\left(\bar{s}_{+}\right)=D_{a c}\left(\bar{s}+i \epsilon^{\prime}\right)$. We ask the reader to carefully discriminate the objects $U_{a b}^{c \pm}(s)$ with $s \in C_{c}$ as introduced in Eq. (13) from the newly introduced object $U_{a b}^{ \pm c}(\bar{s})$ with $\bar{s}$ defined on the real axis only in Eq. (36).

In the following we will show that given the function $\hat{\varsigma}_{a b}(\bar{s})$ only the $D_{a b}(s)$ function can be computed unambiguously. Note that this requires the solution of a linear integral equation since $\Delta \zeta_{a b}(\bar{s})$ requires the knowledge of the $D_{a b}(s)$ function. In order to solve this system it is useful to introduce some notation

$$
\begin{gathered}
\rho_{a b}^{L}(\bar{s})=\sum_{c<a}\left[U_{a c}^{+c}(\bar{s})-U_{a c}^{-c}(\bar{s})\right] \rho_{c b}(\bar{s}) \Theta\left[\left(\mu_{a c}^{A}\right)^{2}<\bar{s}<\hat{\mu}_{b}^{2}\right] \\
\text { for } a>b,
\end{gathered}
$$$$
\rho_{a b}^{L}(\bar{s})=0 \text { for } a \leq b \text { and }
$$$$
\varsigma_{a b}(\bar{s})=0 \text { for } \bar{s}<\left(m_{b}+M_{b}\right)^{2} \text {, }
$$

where $\bar{s}$ is strictly on the real axis. Note that due to the particular form of the anomalous box term $U_{a b}^{\text {box }}(s)$ in Eq. (19) there is no contribution from the latter to $\rho_{a b}^{L}(\bar{s})$. In the general case where the intervals $\left\{\mu_{b}^{2}, \hat{\mu}_{b}^{2}\right\}$ partially overlap some additional notation is useful. We introduce

$$
\begin{aligned}
& \gamma_{a b}(\bar{s})=2 i \sum_{c<a} U_{a c}^{-c}(\bar{s}) \rho_{c b}(\bar{s}) \Theta\left[\mu_{c}^{2}<\bar{s}<\hat{\mu}_{b}^{2}\right], \\
& \bar{\rho}_{a b}^{L}(\bar{s})=\sum_{c} \rho_{a c}^{L}(\bar{s})[1-\gamma(\bar{s})]_{c b}^{-1}, \\
& \bar{\rho}_{a b}^{L}(\bar{s})=0=\gamma_{a b}(\bar{s}) \quad \text { for } \quad a \leq b,
\end{aligned}
$$

where we note that now the box term $U_{a b}^{\text {box }}(s)$ does contribute to the matrix-valued function $\gamma_{a b}(\bar{s})$. Our result (34) and (36) is expressed in the notation (37)-(38) as follows:

$$
\begin{aligned}
D_{a b}(s)= & \delta_{a b}+\int \frac{d \bar{s}}{\pi} \frac{s-\mu^{2}}{\bar{s}-\mu^{2}} \frac{\hat{s}_{a b}(\bar{s})}{\bar{s}-s} \\
& +\sum_{c} \int \frac{d \bar{s}}{\pi} \frac{s-\mu^{2}}{\bar{s}-\mu^{2}} \frac{D_{a c}\left(\bar{s}+i \epsilon^{\prime}\right) \bar{\rho}_{c b}^{L}(\bar{s})}{\bar{s}-s},
\end{aligned}
$$

where all integrals over $\bar{s}$ are on the real axis. The bounds of the integrals are provided by the properties of $\hat{\varsigma}_{a b}(\bar{s})$ and $\bar{\rho}_{c b}^{L}(\bar{s})$ as summarized in Eqs. (37)-(38). We observe a simplification that arises if the intervals $\left\{\mu_{b}^{2}, \hat{\mu}_{b}^{2}\right\}$ are nonoverlapping for different $b$. In this case the term in the third line of Eq. (36) has no effect and thus we find $\bar{\rho}_{a b}^{L}(\bar{s})=\rho_{a b}^{L}(\bar{s})$.

It remains to express $D_{a b}(s)$ in terms of $\hat{\varsigma}_{a b}(s)$ and $\bar{\rho}_{a b}^{L}(s)$. In the nonoverlapping case this is readily achieved by an iteration in the index $b$. At $b=\max$ the second term in Eq. (39) does not contribute as a consequence of the second condition in Eq. (37). In turn we can compute $D_{a b}$ at $b=\max$ for all $a$. In the next step we study $D_{a b}(s)$ at $b=\max -1$, where now the second term in Eq. (39) becomes relevant. However, here only the previously computed $D_{a b}(s)$ at $b=\max$ are needed. This process can be iterated down to the computation of $D_{a 1}(s)$. While this strategy always leads to the correct result it is not very efficient for the following developments.

A more powerful framework can be readily established as follows. We introduce a Green's function $L(x, y)$ via the condition

$\int d y\left[\delta(x-y)-\frac{1}{\pi} \frac{\bar{\rho}^{L}(y)}{x-y-i \epsilon^{\prime}}\right] L(y, z)=\delta(x-z)$,

where we suppress the coupled-channel matrix structure for notational clarity. All objects will be written in the correct order, so that the matrix structure can be reconstructed unambiguously for any identity presented below. The $i \epsilon^{\prime}$ prescription in the definition of $L(x, y)$ in Eq. (40) is inherited from the $i \epsilon^{\prime}$ prescription in Eq. (39). Given the Green's function $D_{a b}(s)$ can be expressed in terms of $\bar{\zeta}_{a b}(s)$ with

$$
\begin{aligned}
D_{a b}(s) & =\delta_{a b}+\int \frac{d \bar{s} s-\mu^{2}}{\pi} \frac{\varsigma_{a b}^{D}(\bar{s})}{\bar{s}-\mu^{2}} \\
\varsigma_{a b}^{D}(s) & =\sum_{c} \int d \bar{s} \frac{s-\mu^{2}}{\bar{s}-\mu^{2}}\left(\hat{\varsigma}_{a c}(\bar{s})+\bar{\rho}_{a c}^{L}(\bar{s})\right) L_{c b}(\bar{s}, s) .
\end{aligned}
$$

The representation (41) does not look very promising for numerical simulations since the Green's function is a highly singular object. However, a closed form can be derived in terms of six analytic matrix functions $u_{n}^{L}(s)$ and $U_{n}^{L}(s)$ with $n=1,2$, 3. The latter are determined by appropriate integrals involving the anomalous spectral weight $\bar{\rho}^{L}(s)$ only. After some algebra we establish the following form for the Green's function: 

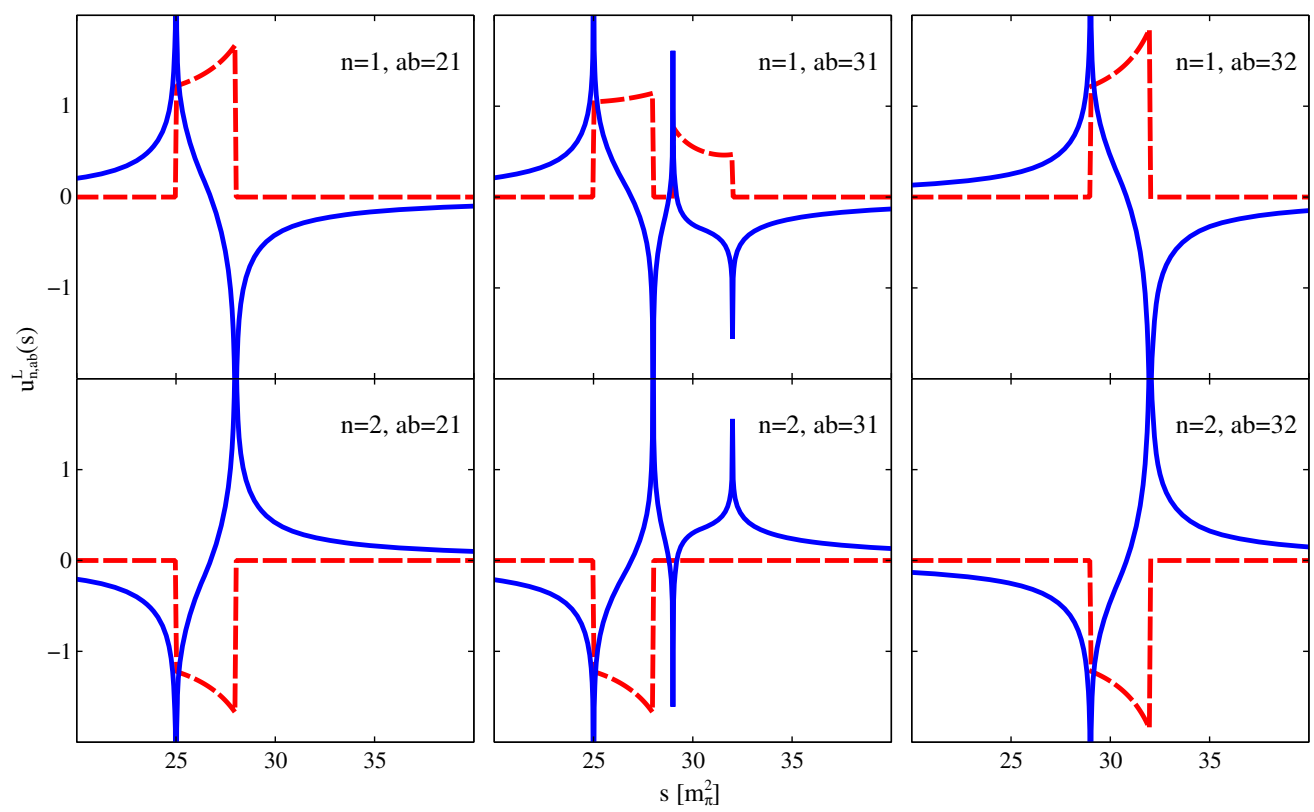

FIG. 11. The nonvanishing functions $u_{n, a b}^{L}(s)$ of Eq. (44) for $n=1,2$ in our schematic model (15). Real and imaginary parts are shown with solid blue and dashed red lines respectively.

$$
L(x, y)=\delta(x-y)+\frac{1}{\pi} \frac{\bar{\rho}^{L}(y)}{x-y-i \epsilon^{\prime}}+\sum_{n=1}^{3} u_{n}^{L}(x) \frac{U_{n}^{L}(x)-U_{n}^{L}(y)}{x-y} \frac{1}{\pi} \bar{\rho}^{L}(y),
$$

where

$$
\begin{aligned}
& u_{1}^{L}(x)=g^{L}(x)-\int \frac{d z}{\pi} \frac{\bar{\rho}^{L}(z) g^{L}(z)}{z-x-i \epsilon^{\prime}}, \quad u_{2}^{L}(x)=h^{L}(x)-\int \frac{d z}{\pi} \frac{\bar{\rho}^{L}(z) h^{L}(z)}{z-x-i \epsilon^{\prime}}, \\
& U^{L}(x)=\int \frac{d z}{\pi} \frac{\bar{\rho}^{L}(z)}{z-x-i \epsilon^{\prime}} \quad u_{3}^{L}(x)=-1, \quad \text { with } \quad g^{L}(x)=U^{L}(x)-\int \frac{d z}{\pi}\left[U^{L}(z)-U^{L}(x)\right] \frac{\bar{\rho}^{L}(z)}{z-x} g^{L}(z), \\
& h^{L}(x)=1-\int \frac{d z}{\pi}\left[U^{L}(z)-U^{L}(x)\right] \frac{\bar{\rho}^{L}(z)}{z-x} h^{L}(z), \\
& U_{1}^{L}(x)=\int \frac{d z}{\pi} \frac{\bar{\rho}^{L}(z)}{z-x}\left[U^{L}(z) \Delta U_{1}^{L}(z, x)+\Delta U_{2}^{L}(z, x)\right], \\
& U_{2}^{L}(x)=-U U^{L}(x)-\int \frac{d z}{\pi} \frac{U^{L}(z) \bar{\rho}^{L}(z)}{z-x}\left[U^{L}(z) \Delta U_{1}^{L}(z, x)+\Delta U_{2}^{L}(z, x)\right], \\
& U_{3}^{L}(x)=\int \frac{d z}{\pi} \frac{\bar{\rho}^{L}(z) g^{L}(z)}{z-x} \Delta U_{1}^{L}(z, x)+\int \frac{d z}{\pi} \frac{\bar{\rho}^{L}(z) h^{L}(z)}{z-x} \Delta U_{2}^{L}(z, x), \quad \text { with } \quad \Delta U_{n}^{L}(z, x)=U_{n}^{L}(z)-U_{n}^{L}(x) .
\end{aligned}
$$

We illustrate the general form of the complex objects $u_{n}^{L}(s)$ and $U_{n}^{L}(s)$ using our schematic model (15). In Fig. 11 and Fig. 12 all elements are shown.

An important property of our result (41) is that the imaginary part of the spectral weight $\varsigma_{a b}^{D}(\bar{s})$ does not vanish in the presence of anomalous threshold effects. This follows from the results (41)-(44). In turn the functions $D_{a b}(s)$ do not satisfy the Schwarz reflection principle with

$$
\begin{aligned}
D_{a b}^{*}(s) & \neq D_{a b}\left(s^{*}\right) \\
\leftrightarrow \quad \operatorname{Im} \hat{\varsigma}_{a b}(\bar{s}) & \neq-\sum_{c} \operatorname{Im}\left[D_{a c}(\bar{s}+i \epsilon) \bar{\rho}_{c b}^{L}(\bar{s})\right],
\end{aligned}
$$

even in the limit with $\epsilon \rightarrow 0$. We emphasize that this is unavoidable in our formulation. Nevertheless we expect that our final reaction amplitudes $T_{a b}^{*}(s)=T_{a b}\left(s^{*}\right)$ will satisfy the Schwarz reflection principle, which plays a crucial role in the derivation of the coupled-channel unitarity condition. 


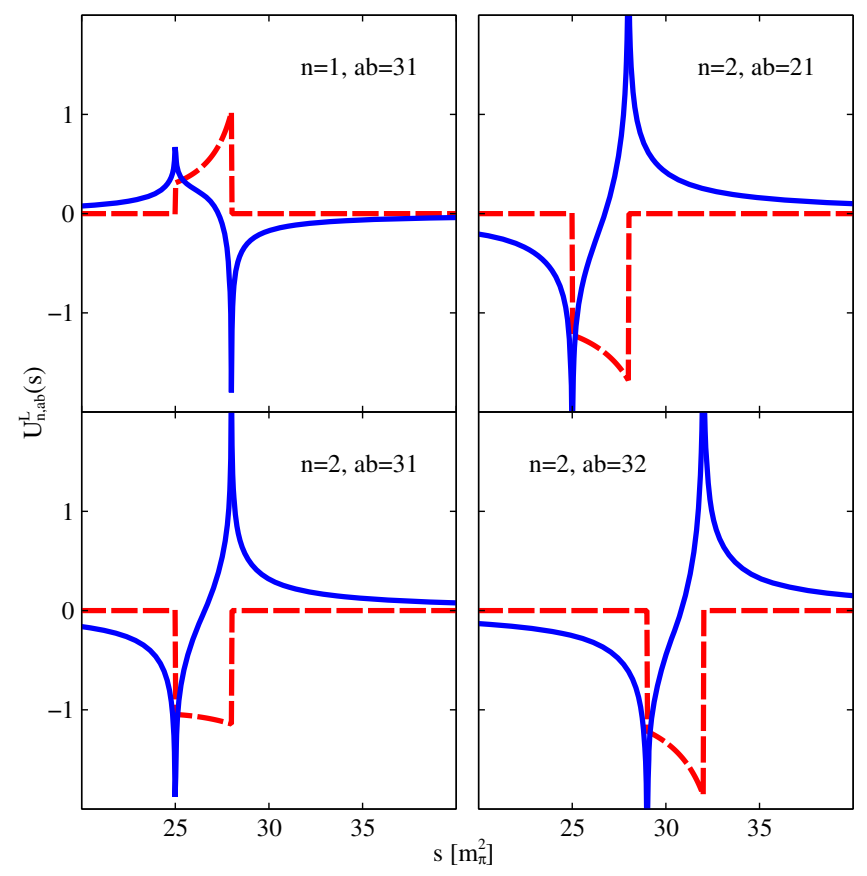

FIG. 12. The nonvanishing functions $U_{n, a b}^{L}(s)$ of Eq. (44) in our schematic model (15). Note the relation $U_{3,31}^{L}(s)=U_{1,31}^{L}(s)$. Real and imaginary parts are shown with solid blue and dashed red lines respectively.

Note that Eq. (45) should not be surprising since we have already discussed that the anomalous box term $U_{a b}^{\text {box }}(\bar{s})$ is at odds with Eq. (17). The relation (45) can be confirmed explicitly upon an expansion of the $D$ function in powers of the generalized potential. At second order there is a term that confirms Eq. (45).

We continue with the $B$ function, which is given by

$$
B_{a b}(s)=\sum_{c} \int_{C_{c}} \frac{d \bar{s}}{\pi} \frac{s-\mu^{2}}{\bar{s}-\mu^{2}} \frac{\varsigma_{a c}(\bar{s})}{\bar{s}-s} U_{c b}(\bar{s}),
$$

in terms of the spectral weight $\varsigma_{a c}(s)$ and the generalized potential $U_{a c}(s)$. Again we perform the limit $\epsilon \rightarrow 0$, in which the complex contours $C_{c}$ all approach the real axis.

Following the decomposition of the contour lines $C_{c}$ as introduced in our study of the $D$ function in Eq. (34) we readily derive the corresponding form

$$
\begin{aligned}
B_{a b}(s)= & \sum_{c} \int_{\left(m_{c}+M_{c}\right)^{2}}^{\infty} \frac{d \bar{s} s-\mu^{2}}{\pi} \frac{\hat{\beta}_{a b}^{c}(\bar{s})}{\bar{s}-\mu^{2}} \overline{\bar{s}-s} \\
& +\sum_{c} \int_{\mu_{c}^{2}}^{\hat{\mu}_{c}^{2}} \frac{d \bar{s}}{\pi} \frac{s-\mu^{2}}{\bar{s}-\mu^{2}} \frac{\Delta \beta_{a b}^{c}(\bar{s})}{\bar{s}-s},
\end{aligned}
$$

with

$$
\begin{aligned}
\hat{\beta}_{a b}^{c}(\bar{s})= & \varsigma_{a c}^{+}(\bar{s}) U_{c b}^{+c}(\bar{s}) \Theta\left(\hat{\mu}_{c}^{2}-\bar{s}\right) \\
& +\varsigma_{a c}^{-}(\bar{s}) U_{c b}^{-c}(\bar{s}) \Theta\left(\bar{s}-\hat{\mu}_{c}^{2}\right), \\
\Delta \beta_{a b}^{c}(\bar{s})= & \varsigma_{a c}^{-}(\bar{s}) U_{c b}^{-c}(\bar{s})-\varsigma_{a c}^{+}(\bar{s}) U_{c b}^{+c}(\bar{s}),
\end{aligned}
$$

where the integrals over $\bar{s}$ in Eq. (47) are strictly on the real axis. Again modulo contributions in Eq. (46) from vertical parts of the contours $C_{c}$ both representations (46) and (47) are identical at any finite $\epsilon$. In Eq. (47) we apply the convenient notation $\varsigma_{a c}^{ \pm}(\bar{s})$ and $U_{c b}^{ \pm c}(\bar{s})$ introduced already in Eqs. (35)-(36).

In the following we will express the spectral weights $\hat{\beta}_{a b}^{c}(\bar{s})$ and $\Delta \beta_{a b}^{c}(\bar{s})$ in terms of $\hat{\zeta}_{a b}(\bar{s})$ and $\Delta \varsigma_{a b}(\bar{s})$. For this we will have to consider the limit $\epsilon \rightarrow 0$ again, in which we find

$$
\hat{\beta}_{a b}^{c}(\bar{s})=\hat{\kappa}_{a c}(\bar{s}) U_{c b}(\bar{s}) \text { for } \bar{s}>\left(m_{c}+M_{c}\right)^{2},
$$

where the generalized potential $U_{c b}(\bar{s})$ is evaluated strictly on the real axis. It is important to realize that $U_{c b}(\bar{s})$ is always needed in between the upper and lower anomalous cut lines (dashed lines in Fig. 2) even after the limit $\epsilon \rightarrow 0$ has been performed. For the anomalous spectral weight $\Delta \beta_{a b}^{c}(\bar{s})$ we derive three distinct contributions

$$
\Delta \beta_{a b}^{c}(\bar{s})= \begin{cases}\Delta \varsigma_{a c}(\bar{s}) U_{c b}^{-c}(\bar{s})+\varsigma_{a c}^{+}(\bar{s})\left[U_{c b}^{-c}(\bar{s})-U_{c b}^{+c}(\bar{s})\right] & b>c, \\ \Delta \varsigma_{a c}(\bar{s}) U_{c b}(\bar{s}) & b \leq c, \\ \Theta\left[\bar{s}-\mu_{c}^{2}\right] \Theta\left[\hat{\mu}_{b}^{2}-\bar{s}\right] \Delta \varsigma_{a c}(\bar{s})\left[U_{c b}^{-b}(\bar{s})-U_{c b}^{+b}(\bar{s})\right] & b<c,\end{cases}
$$

which contribute depending on the various cases $b>c, b<c$ or $b=c$. We express our results (47)-(49) in the notation (37)-(38) as follows:

$$
\begin{aligned}
B_{a b}(s)= & \sum_{c} \int_{\left(m_{c}+M_{c}\right)^{2}}^{\infty} \frac{d \bar{s} s-\mu^{2}}{\pi} \frac{\hat{s}_{a c}(\bar{s})}{\bar{s}-\mu^{2}} U_{c b}(\bar{s})+\sum_{c \geq b, d} \int \frac{d \bar{s}}{\pi} \frac{s-\mu^{2}}{\bar{s}-\mu^{2}} \frac{D_{a d}\left(\bar{s}+i \epsilon^{\prime}\right) \bar{\rho}_{d c}^{L}(\bar{s})}{\bar{s}-s} U_{c b}(\bar{s}) \\
& +\sum_{c<b, d} \int \frac{d \bar{s}}{\pi} \frac{s-\mu^{2}}{\bar{s}-\mu^{2}} \frac{D_{a d}\left(\bar{s}+i \epsilon^{\prime}\right) \bar{\rho}_{d c}^{L}(\bar{s})}{\bar{s}-s} U_{c b}^{-c}(\bar{s})+\sum_{c<b} \int_{\mu_{c}^{2}}^{\hat{\mu}_{c}^{2}} \frac{d \bar{s} s-\mu^{2}}{\bar{s}} \frac{\hat{s}_{a c}^{+}(\bar{s})}{\bar{s}-\mu^{2}}\left[U_{c b}^{-c}(\bar{s})-U_{c b}^{+c}(\bar{s})\right] \\
& +\sum_{c>b, d} \int_{\mu_{c}^{2}}^{\hat{\mu}_{b}^{2}} \frac{d \bar{s} s-\mu^{2}}{\pi} \frac{D_{a d}(\bar{s}+i \epsilon) \bar{\rho}_{d c}^{L}(\bar{s})}{\bar{s}-\mu^{2}}\left[U_{c b}^{-b}(\bar{s})-U_{c b}^{+b}(\bar{s})\right],
\end{aligned}
$$


where all integrals over $\bar{s}$ are on the real axis. The bounds of the integrals are provided directly or by the properties of $\bar{\rho}_{c b}^{L}(\bar{s})$ as summarized in Eqs. (37)-(38).

We point out an important subtlety. While in the previous section we managed to express $D_{a b}(s)$ in terms of $\hat{\varsigma}_{a b}(s)$, this is not possible for $B_{a b}(s)$ : the term in Eq. (50) involving $\hat{\zeta}_{a c}^{+}(s)$ is required at $s<\left(m_{c}+M_{c}\right)^{2}$ outside the domain where $\hat{\varsigma}_{a c}(s)$ was introduced in Eq. (34). However, since $\hat{\varsigma}_{a c}(s)=\hat{\varsigma}_{a c}^{+}(s)$ for $s<\hat{\mu}_{c}^{2}$ we can simply extend the domain of $\hat{\varsigma}_{a c}(s)$ where it is defined.

It is useful to provide a further rewriting of the $B$ function that uses the spectral weight of the $D$ function $\varsigma_{a b}^{D}(\bar{s})$. We find

$$
\begin{aligned}
B_{a b}(s)= & \sum_{c} \int \frac{d \bar{s}}{\pi} \frac{s-\mu^{2}}{\bar{s}-\mu^{2}} \frac{\varsigma_{a c}^{D}(\bar{s})}{\bar{s}-s} U_{c b}(\bar{s}) \\
& -\sum_{c<b} \int_{\mu_{c}^{2}}^{\hat{\mu}_{c}^{2}} \frac{d \bar{s} s-\mu^{2}}{\pi} \frac{\varsigma_{a c}^{D}(\bar{s})-\hat{\varsigma}_{a c}(\bar{s})}{\bar{s}-\mu^{2}} \Delta U_{c b}(\bar{s}) \\
& -\sum_{c<b} \int_{\mu_{c}^{2}}^{\hat{\mu}_{c}^{2}} \frac{d \bar{s}}{\pi} \frac{s-\mu^{2}}{\bar{s}-\mu^{2}} \frac{\hat{s}_{a c}^{+}(\bar{s})}{\bar{s}-s} \Delta U_{c b}(\bar{s}) \\
& -\sum_{c>b} \int_{\mu_{c}^{2}}^{\hat{\mu}_{b}^{2}} \frac{d \bar{s}}{\pi} \frac{\mu^{2}}{\bar{s}-\mu^{2}} \frac{\varsigma_{a c}^{D}(\bar{s})-\hat{\varsigma}_{a c}(\bar{s})}{\bar{s}-s} \Delta U_{c b}(\bar{s}),
\end{aligned}
$$

with

$$
\begin{aligned}
\Delta U_{a b}(\bar{s})= & \Theta[a-b]\left[U_{a b}^{+b}(\bar{s})-U_{a b}^{-b}(\bar{s})\right] \\
& +\Theta[b-a]\left[U_{a b}^{+a}(\bar{s})-U_{a b}^{-a}(\bar{s})\right],
\end{aligned}
$$

where we recall the particular notation $U_{a b}^{ \pm c}(\bar{s})$ as introduced in Eq. (36).

It should not come as a surprise that like the $D$ function the $B$ function also does not satisfy the Schwarz reflection principle with

$$
B_{a b}^{*}(s) \neq B_{a b}\left(s^{*}\right),
$$

even in the limit with $\epsilon \rightarrow 0$. This is readily verified. If expanded to second order in powers of the generalized potential there must be an anomalous contribution with Eq. (53) that cancels the effect of the anomalous box contribution (21). This is so since by construction the full reaction amplitude was constructed to satisfy Eq. (17) at least to second order in a perturbative expansion.

\section{LINEAR INTEGRAL EQUATION ON REAL CONTOURS}

In the previous two sections we have expressed the $D_{a b}(s)$ and $B_{a b}(s)$ functions in terms of the spectral weight $\hat{\varsigma}_{a b}(\bar{s})$. In this section we wish to establish a set of linear integral equations for $\hat{\varsigma}_{a b}(\bar{s})$ given a generalized potential $U_{a b}(s)$. These equations will serve as an alternative formulation of Eq. (32), which is suitable for numerical simulations. After some necessary steps detailed in this section we will arrive at an integral equation of the form

$$
\begin{aligned}
\hat{\varsigma}(s)= & -\hat{U}(s) \rho(s)+\sum_{m, n=1}^{3} \int \frac{d \bar{s} s-\mu^{2}}{\pi} \frac{\mu^{2}}{\bar{s}}(\bar{s}) u_{m}^{L}(\bar{s}) \\
& \times \frac{\hat{U}_{m n}(\bar{s})-\hat{U}_{m n}(s)}{\bar{s}-s} u_{n}^{R}(s),
\end{aligned}
$$

in terms of a set of analytic matrix functions $\hat{U}(s)$, $\hat{U}_{m n}(s)$ and $u_{m}^{L}(s), u_{m}^{R}(s)$. The latter will be expressed in terms of the generalized potential $U(s)$. Note that in Eq. (54) we suppressed the coupled-channel indices. The terms are ordered properly so that the coupledchannel structure is correctly implied by standard matrix multiplication rules.

How can we cast the contour integral equation (32) into an integral equation (54) where all integrals are strictly on the real axis? Several steps are required. The first task is to express $\varsigma_{a b}^{ \pm}(s)$ in terms of $\varsigma_{a b}(s)$ only. We begin with the consideration of $\varsigma_{a b}^{-}(s)$, which we evaluate according to the second identity in Eq. (23) with

$\varsigma_{a b}(s)=\sum_{d}\left[B_{a d}^{b-}(s)-\sum_{c} D_{a c}^{b-}(s) U_{c d}^{b-}(s)\right] \rho_{d b}(s)$ at $s \in C_{b}$,

for which we consider the contour limit $\epsilon \rightarrow 0$ in Fig. 3. The reaction amplitude $T_{c d}^{b-}(\bar{s})$ in Eq. (23) is expressed in terms of the $B_{a b}^{b-}(\bar{s})$ function as evaluated in Eq. (50). Similarly for the required $D_{a c}^{b-}(\bar{s})$ function we use Eq. (39). Then for $\epsilon \rightarrow 0$ and $s>\hat{\mu}_{b}^{2}$ we find 


$$
\begin{aligned}
\hat{\varsigma}_{a b}(s)= & \varsigma_{a b}^{-}(s)=-\sum_{c} U_{a c}(s) \rho_{c b}(s)+\sum_{c, d} \int \frac{d \bar{s}}{\pi} \frac{s-\mu^{2}}{\bar{s}-\mu^{2}} \frac{\hat{s}_{a c}(\bar{s})}{\bar{s}-s}\left[U_{c d}(\bar{s})-U_{c d}(s)\right] \rho_{d b}(s) \\
& +\sum_{c \geq b, d, e} \int \frac{d \bar{s}}{\pi} \frac{s-\mu^{2}}{\bar{s}-\mu^{2}} \frac{D_{a e}\left(\bar{s}+i \epsilon^{\prime}\right) \bar{\rho}_{e c}^{L}(\bar{s})}{\bar{s}-s}\left[U_{c d}(\bar{s})-U_{c d}(s)\right] \rho_{d b}(s) \\
& +\sum_{c<b, d, e} \int \frac{d \bar{s}}{\pi} \frac{s-\mu^{2}}{\bar{s}-\mu^{2}} \frac{D_{a e}\left(\bar{s}+i \epsilon^{\prime}\right) \bar{\rho}_{e c}^{L}(\bar{s})}{\bar{s}-s}\left[U_{c d}^{-c}(\bar{s})-U_{c d}(s)\right] \rho_{d b}(s) \\
& -\sum_{c<b, d} \int_{\mu_{c}^{2}} \frac{\hat{\mu}_{c}^{2}}{\pi} \frac{d \bar{s}-\mu^{2}}{\bar{s}-\mu^{2}} \frac{\hat{\varsigma}_{a c}^{+}(\bar{s})}{\bar{s}-s+i \epsilon^{\prime}} \Delta U_{c d}(\bar{s}) \rho_{d b}(s)-\sum_{c>b, d, e} \int_{\mu_{c}^{2}} \frac{\hat{\mu}_{b}^{2}}{\pi} \frac{\bar{s}}{\bar{s}-\mu^{2}} \frac{D_{a e}(\bar{s}+i \epsilon) \bar{\rho}_{e c}^{L}(\bar{s})}{\bar{s}-s+i \epsilon^{\prime}} \Delta U_{c d}(\bar{s}) \rho_{d b}(s),
\end{aligned}
$$

where we again use the particular notations $\Delta U_{a b}(\bar{s})$ and $U_{a b}^{ \pm c}(\bar{s})$ as introduced in Eqs. (52) and (36). We emphasize that $s$ and $\bar{s}$ in Eq. (56) are strictly on the real axis as is implied by the limit $\epsilon \rightarrow 0$. It is important to realize that $U_{c b}(s)$ is always evaluated in between the upper and lower anomalous cut lines (the dashed lines in Fig. 2).

We continue with the more complicated case $\varsigma_{a b}^{+}(s)$ at $s<\hat{\mu}_{b}^{2}$. This time we start with the first identity in Eq. (23) with

$\varsigma_{a b}(s)=\sum_{d}\left[B_{a d}^{b+}(s)-\sum_{c} D_{a c}^{b+}(s) U_{c d}^{b+}(s)\right] \rho_{d b}(s)$ at $s \in C_{b}$,

and again consider the contour limit $\epsilon \rightarrow 0$. For an evaluation of $\varsigma_{a b}^{+}(s)$ we will need the $D_{a c}(s)$ and $B_{a b}(s)$ functions evaluated slightly above the contour $C_{b}$ with $s \in C_{b}$. The latter cannot be deduced directly from the results of the previous two sections. This is so because sometimes the functions are required in between two righthand cut lines, for which the results (39) and (50) cannot be applied. Some intermediate steps are required. Consider first the $B_{a b}^{b+}(s)$ term [the first contribution in Eq. (57)]. In the limit $\epsilon \rightarrow 0$ we can derive

$$
\begin{aligned}
B_{a d}^{b+}(s)= & B_{a d}\left(s+i \epsilon^{\prime}\right)-\sum_{c<b} 2 i \varsigma_{a c}^{+}(s)\left(U_{c d}(s)\right. \\
& \left.-\Delta U_{c d}(s)\right) \Theta\left[s-\mu_{b}^{2}\right] \Theta\left[\hat{\mu}_{c}^{2}-s\right]
\end{aligned}
$$

where we consider $s$ in Eq. (58) to be strictly real again. For the required kinematics with $s<\hat{\mu}_{b}^{2}$ and $\epsilon \rightarrow 0$ it follows that

$$
\begin{aligned}
B_{a b}^{b+}(s)= & \sum_{c<b} \int \frac{d \bar{s}}{\pi} \frac{s-\mu^{2}}{\bar{s}-\mu^{2}} \frac{\hat{\varsigma}_{a c}(\bar{s})}{\bar{s}-s+i \epsilon_{c b}^{\prime}} U_{c b}(\bar{s})+\sum_{c \geq b} \int \frac{d \bar{s}}{\pi} \frac{s-\mu^{2}}{\bar{s}-\mu^{2}} \frac{\hat{\varsigma}_{a c}(\bar{s})}{\bar{s}-s-i \epsilon^{\prime}} U_{c b}(\bar{s}) \\
& +\sum_{c \geq b, d} \int \frac{d \bar{s}}{\pi} \frac{s-\mu^{2}}{\bar{s}-\mu^{2}} \frac{D_{a d}\left(\bar{s}+i \epsilon^{\prime}\right) \bar{\rho}_{d c}^{L}(\bar{s})}{\bar{s}-s-i \epsilon^{\prime}} U_{c b}(\bar{s})+\sum_{c<b, d} \int \frac{d \bar{s}}{\pi} \frac{s-\mu^{2}}{\bar{s}-\mu^{2}} \frac{D_{a d}\left(\bar{s}+i \epsilon^{\prime}\right) \bar{\rho}_{d c}^{L}(\bar{s})}{\bar{s}-s-i \epsilon^{\prime}} U_{c b}^{-c}(\bar{s}) \\
& -\sum_{c<b} \int_{\mu_{c}^{2}}^{\hat{\mu}_{c}^{2}} \frac{d \bar{s}}{\pi} \frac{s-\mu^{2}}{\bar{s}-\mu^{2}} \frac{\hat{s}_{a c}^{+}(\bar{s})}{\bar{s}-s+i \epsilon_{c b}^{\prime}} \Delta U_{c b}(\bar{s})-\sum_{c>b, d} \int_{\mu_{c}^{2}}^{\hat{\mu}_{b}^{2}} \frac{d \bar{s}}{\pi} \frac{s-\mu^{2}}{\bar{s}-\mu^{2}} \frac{D_{a d}\left(\bar{s}+i \epsilon^{\prime}\right) \bar{\rho}_{d c}^{L}(\bar{s})}{\bar{s}-s-i \epsilon^{\prime}} \Delta U_{c b}(\bar{s})
\end{aligned}
$$

where we applied Eq. (58) in combination with Eq. (50). We point out the different prescriptions $s \pm i \epsilon^{\prime}$ in the various terms in Eq. (59) with

$$
\epsilon_{c b}^{\prime}= \begin{cases}+\epsilon^{\prime} & \text { for } \mu_{b}^{2}<s<\hat{\mu}_{c}^{2} \\ -\epsilon^{\prime} & \text { otherwise. }\end{cases}
$$

The change in prescription is caused by the terms on the right-hand side of Eq. (58).

We proceed with the second term in Eq. (57). Here we need to evaluate $D_{a c}^{b+}(s) U_{c b}^{b+}(s)$ in the limit $\epsilon \rightarrow 0$. Progress is based on the identity

$$
D_{a c}^{b+}(s)=D_{a c}\left(s+i \epsilon^{\prime}\right)+ \begin{cases}-2 i \varsigma_{a c}^{+}(s) \Theta\left[s-\mu_{b}^{2}\right] \Theta\left[\hat{\mu}_{c}^{2}-s\right] & \text { for } b>c, \\ 0 & \text { for } b \leq c,\end{cases}
$$


which again follows in the limit $\epsilon \rightarrow 0$. From Eq. (61) it now follows that

$\sum_{c \geq b} D_{a c}^{b+}(s) U_{c b}^{b+}(s)=\sum_{c \geq b} D_{a c}\left(s+i \epsilon^{\prime}\right) U_{c b}(s)$,
$\sum_{c<b} D_{a c}^{b+}(s) U_{c b}^{b+}(s)=U_{a b}(s)+\sum_{c<b} \int \frac{d \bar{s}}{\pi} \frac{s-\mu^{2}}{\bar{s}-\mu^{2}} \frac{\hat{\varsigma}_{a c}(\bar{s})}{\bar{s}-s+i \epsilon_{c b}^{\prime}} U_{c b}(s)+\sum_{c<b, d} \int \frac{d \bar{s}}{\pi} \frac{s-\mu^{2}}{\bar{s}-\mu^{2}} \frac{D_{a d}\left(\bar{s}+i \epsilon^{\prime}\right) \bar{\rho}_{d c}^{L}(\bar{s})}{\bar{s}-s-i \epsilon^{\prime}} U_{c b}(s)$,

where we assumed $\left(m_{b}+M_{b}\right)^{2}<s<\hat{\mu}_{b}^{2}$ and $\epsilon \rightarrow 0$. Combining our results (59) and (62) we arrive at the desired expression

$$
\begin{aligned}
\hat{\varsigma}_{a b}(s)= & \varsigma_{a b}^{+}(s)=-\sum_{c} U_{a c}(s) \rho_{c b}(s)+\sum_{c, d} \int \frac{d \bar{s}}{\pi} \frac{s-\mu^{2}}{\bar{s}-\mu^{2}} \frac{\hat{s}_{a c}(\bar{s})}{\bar{s}-s}\left[U_{c d}(\bar{s})-U_{c d}(s)\right] \rho_{d b}(s) \\
& +\sum_{c, d, e} \int \frac{d \bar{s}}{\pi} \frac{s-\mu^{2}}{\bar{s}-\mu^{2}} \frac{D_{a e}\left(\bar{s}+i \epsilon^{\prime}\right) \bar{\rho}_{e c}^{L}(\bar{s})}{\bar{s}-s}\left[\bar{U}_{c d}(\bar{s})-\bar{U}_{c d}(s)\right] \rho_{d b}(s)-\sum_{c<b} \int_{\mu_{c}^{2}}^{\hat{\mu}_{c}^{2}} \frac{d \bar{s}}{\pi} \frac{s-\mu^{2}}{\bar{s}-\mu^{2}} \frac{\varsigma_{a c}^{+}(\bar{s})}{\bar{s}-s+i \epsilon_{c b}^{\prime}} \Delta U_{c d}(\bar{s}) \rho_{d b}(s) \\
& -\sum_{c>b, d, e} \int_{\mu_{c}^{2}}^{\hat{\mu}_{b}^{2}} \frac{d \bar{s}}{\pi} \frac{s-\mu^{2}}{\bar{s}-\mu^{2}} \frac{D_{a e}(\bar{s}+i \epsilon) \bar{\rho}_{e c}^{L}(\bar{s})}{\bar{s}-s-i \epsilon^{\prime}} \Delta U_{c d}(\bar{s}) \rho_{d b}(s), \quad \text { for }\left(m_{b}+M_{b}\right)^{2}<s<\hat{\mu}_{b}^{2}, \\
\bar{U}_{a b}(\bar{s})= & U_{a b}(\bar{s}) \quad \text { for } a>b, \quad \bar{U}_{a b}(\bar{s})=U_{a b}\left(\bar{s}-i \epsilon^{\prime}\right) \quad \text { for } a<b,
\end{aligned}
$$

where we exploited the corresponding prescription changes in Eqs. (59) and (62) that are related to the first line in Eq. (63). Again we use that $U_{c d}\left(s-i \epsilon^{\prime}\right)=U_{c d}(s)$ for $s>\left(m_{d}+M_{d}\right)^{2}$ and $c<d$. We point out the formal similarity of Eq. (63) with our result (56) derived previously at $s>\hat{\mu}_{b}^{2}$ only. With the exception of the prescription in the last two terms the expressions are identical. Moreover, since that prescription is relevant only for $s<\hat{\mu}_{b}^{2}$ in those terms we arrive at one of the cornerstones of this section

$$
\begin{aligned}
& \hat{\boldsymbol{S}}_{a b}(s)=-\sum_{c} U_{a c}(s) \rho_{c b}(s)+\sum_{c, d} \int \frac{d \bar{s}}{\pi} \frac{s-\mu^{2}}{\bar{s}-\mu^{2}} \frac{\hat{\boldsymbol{S}}_{a c}(\bar{s})}{\bar{s}-s}\left[U_{c d}(\bar{s})-U_{c d}(s)\right] \rho_{d b}(s) \\
& +\sum_{c, d, e} \int \frac{d \bar{s}}{\pi} \frac{s-\mu^{2}}{\bar{s}-\mu^{2}} \frac{D_{a e}\left(\bar{s}+i \epsilon^{\prime}\right) \bar{\rho}_{e c}^{L}(\bar{s})}{\bar{s}-s}\left[\bar{U}_{c d}(\bar{s})-\bar{U}_{c d}(s)\right] \rho_{d b}(s) \\
& -\sum_{c<b, d} \int_{\mu_{c}^{2}}^{\hat{\mu}_{c}^{2}} \frac{d \bar{s}}{\pi} \frac{s-\mu^{2}}{\bar{s}-\mu^{2}} \frac{\varsigma_{a c}^{+}(\bar{s})}{\bar{s}-s+i \epsilon^{\prime}} \Delta U_{c d}(\bar{s}) \rho_{d b}(s)-\sum_{c>b, d, e} \int_{\mu_{c}^{2}}^{\hat{\mu}_{b}^{2}} \frac{d \bar{s}}{\pi} \frac{s-\mu^{2}}{\bar{s}-\mu^{2}} \frac{D_{a e}(\bar{s}+i \epsilon) \bar{\rho}_{e c}^{L}(\bar{s})}{\bar{s}-s-i \epsilon^{\prime}} \Delta U_{c d}(\bar{s}) \rho_{d b}(s),
\end{aligned}
$$

which is valid for any $s>\left(m_{b}+M_{b}\right)^{2}$.

It remains to rewrite our result (64) into a more practical form. This requires three steps. First we multiply Eq. (64) by the pseudoinverse of the phase-space matrix

$$
\begin{aligned}
\hat{N}_{a b}(s)= & U_{a b}(s)+\sum_{c, d} \int \frac{d \bar{s} s-\mu^{2}}{\pi} \frac{\hat{N}_{a c}(\bar{s})}{\bar{s}-\mu^{2}} \hat{\rho}_{c d}(\bar{s})\left[U_{d b}(\bar{s})-U_{d b}(s)\right]-\sum_{c, d} \int \frac{d \bar{s} s-\mu^{2}}{\pi} \frac{D_{a c}\left(\bar{s}+i \epsilon^{\prime}\right) \bar{\rho}_{c d}^{L}(\bar{s})}{\bar{s}-\mu^{2}}\left[\bar{U}_{d b}(\bar{s})-\bar{U}_{d b}(s)\right] \\
& -\sum_{c} \int \frac{d \bar{s} s-\mu^{2}}{\pi} \frac{\hat{N}_{a c}(\bar{s}) \rho_{c b}^{R}(\bar{s})}{\bar{s}-\mu^{2}}+\sum_{c>d>b} \int \frac{d \bar{s} s-\mu^{2} D_{a c}\left(\bar{s}+i \epsilon^{\prime}\right) \bar{\rho}_{c d}^{L}(\bar{s}) \Delta U_{d b}(\bar{s})}{\pi-\epsilon^{\prime}}
\end{aligned}
$$

where the result is expressed in terms of the more convenient building blocks

$$
\begin{aligned}
& \hat{\varsigma}_{a b}(s)=-\sum_{c} \hat{N}_{a c}(s) \hat{\rho}_{c b}(s), \quad \rho_{a b}^{R}(s)=0 \quad \text { for } a>b, \\
& \rho_{a b}^{R}(s)=\sum_{c} \rho_{a c}(s) \Delta U_{c b}(s) \Theta\left[s-\left(\mu_{a b}^{A}\right)^{2}\right] \Theta\left[\hat{\mu}_{a}^{2}-s\right] \quad \text { for } b>a, \\
& \hat{\rho}_{a b}(s)=\rho_{a b}(s) \quad \text { for } s>\left(m_{a}+M_{a}\right)^{2} ; \quad \text { otherwise } \hat{\rho}_{a b}(s)=0 .
\end{aligned}
$$




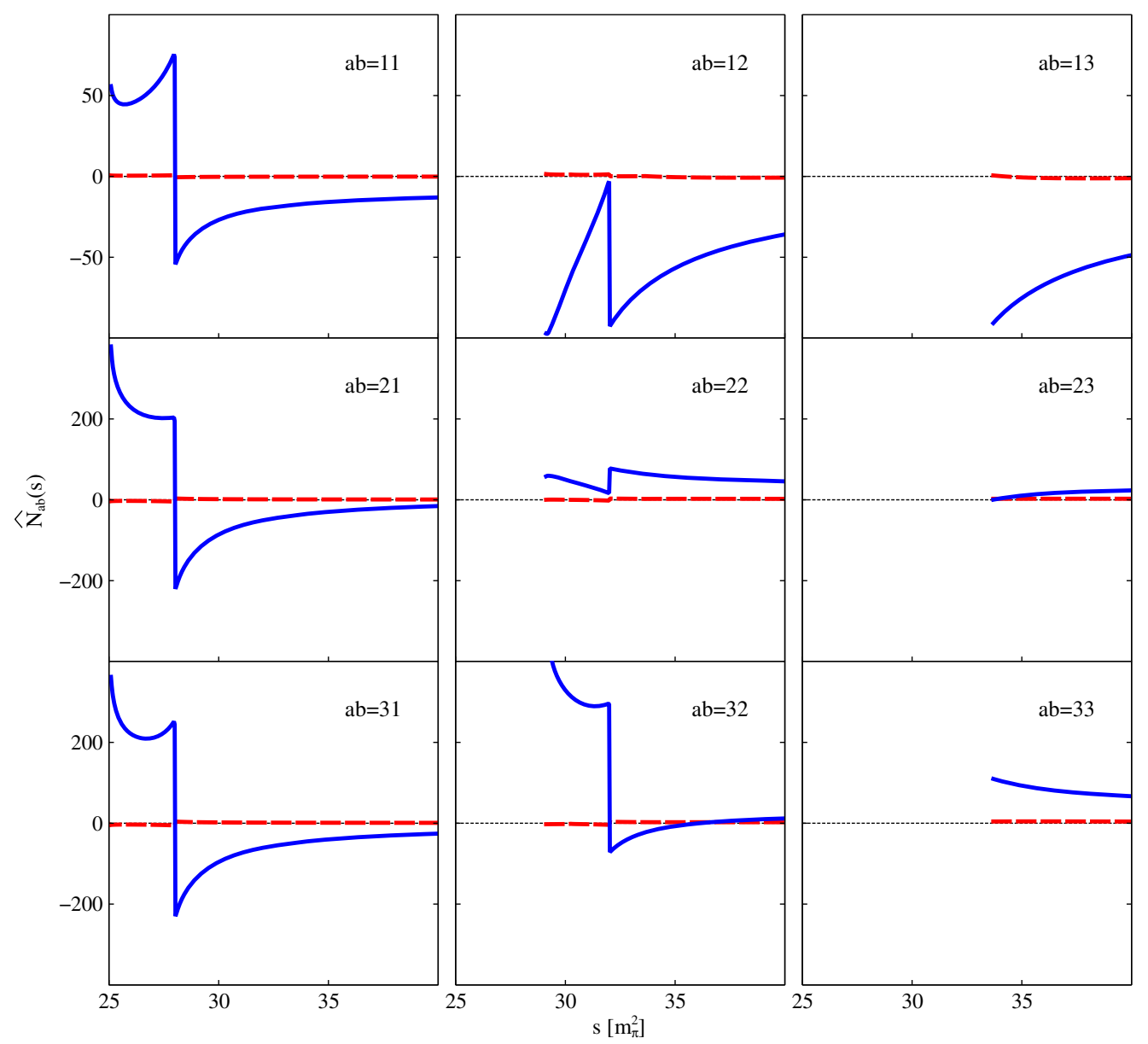

FIG. 13. The functions $\hat{N}_{a b}(s)$ of Eqs. (65), (66), and (78) for $s>\mu_{b}^{2}$ or $s>\left(m_{b}+M_{b}\right)^{2}$ in our schematic model (15). Real and imaginary parts are shown with solid blue and dashed red lines respectively.

The integrals over $\bar{s}$ in Eq. (65) are strictly on the real axis. The integration domains for the various contributions are implied by the region where their integrands are zero as summarized in Eqs. (37) and (66). Note that $\hat{N}_{a b}(s)$ is given by Eq. (65) for $s>\left(m_{b}+M_{b}\right)^{2}$ only where the pseudoinverse of the phase-space matrix $\hat{\rho}_{a b}(s)$ is defined unambiguously. From Eq. (32) it follows that $\hat{N}_{a b}(s)$ inherits the cut lines of the generalized potential $U_{c b}(s)$ for any $c$ only. The cut lines of the phase-space functions are not present in $\hat{N}_{a b}(s)$. This implies that $\hat{N}_{a b}(s)$ has a unique analytic continuation from $s>\left(m_{b}+M_{b}\right)^{2}$ down to $s>\mu_{b}^{2}$, but only as long as we keep $\epsilon$ finite in the contours of Fig. 3. Note that the region $\mu_{b}^{2}<s<\left(m_{b}+M_{b}\right)^{2}$ is accessed by $\hat{N}_{a b}(s)$ in the third line of Eq. (65). Could it be justified to use Eq. (65) at a $s<\left(m_{b}+M_{b}\right)^{2}$ for which it was not derived? We will return to this issue further below.

We exemplify the form of the auxiliary functions $\hat{N}_{a b}(s)$ with our model (15). In Fig. 13 the complex functions are shown in the domain $s>\mu_{b}^{2}$ or $s>\left(m_{b}+M_{b}\right)^{2}$ only as needed for the evaluation of the functions $B_{a b}(s)$ in Eqs. (50) and (59). It is important to note that the latter do depend on the choice of the return point $\hat{\mu}_{b}$. The functions $\hat{N}_{a b}(s)$ are discontinuous at the return points $s=\hat{\mu}_{b}^{2}$.

In the following we perform a further simplification of Eq. (65) valid at $s>\left(m_{b}+M_{b}\right)^{2}$. Our targets are the terms in the last two lines. We begin with the very last term, for which we obtain for $c>b$

$$
\begin{aligned}
\sum_{d>b} \int \frac{d \bar{s}}{\pi} \frac{s-\mu^{2}}{\bar{s}-\mu^{2}} \frac{D_{a c}\left(\bar{s}+i \epsilon^{\prime}\right) \bar{\rho}_{c d}^{L}(\bar{s}) \Delta U_{d b}(\bar{s})}{\bar{s}-s-i \epsilon^{\prime}} & =X_{a b}^{(c>b)}(s)-\sum_{d>c} \int \frac{d \bar{s} s-\mu^{2}}{\pi} \frac{s_{a d}^{D}(\bar{s})}{\bar{s}-\mu^{2}}\left[X_{d b}^{(c>b)}(\bar{s})-X_{d b}^{(c>b)}(s)\right], \\
\text { with } \quad X_{a b}^{(c>b)}(s) & =\int_{\hat{\mu}_{b}^{2}}^{\hat{\mu}_{c}^{2}} \frac{d \bar{s}}{\pi} \frac{s-\mu^{2}}{\bar{s}-\mu^{2}} \frac{\bar{\rho}_{a c}^{L}(\bar{s}) \Delta U_{c b}(\bar{s})}{\bar{s}-s-i \epsilon^{\prime}} \Theta\left[\bar{s}-\left(\mu_{a c}^{A}\right)^{2}\right],
\end{aligned}
$$


where we made the integration domain for the integral representation of $X_{a b}^{(c>b)}(s)$ explicit. This implies that $X_{a b}^{(c>b)}(s)$ is analytic for $s>\hat{\mu}_{c}^{2}>\left(m_{b}+M_{b}\right)^{2}$ and $s<\operatorname{Max}\left(\hat{\mu}_{b}^{2}, \mu_{a c}^{A}\right)$.

The result (67) is useful since it shows that the net effect of this last term is a renormalization of the generalized potential of the form

$$
\begin{aligned}
& U_{a b}^{\mathrm{eff}}(s)=U_{a b}(s)+\sum_{a>c>b} X_{a b}^{(c)}(s) \quad \text { and } \\
& \bar{U}_{a b}^{\mathrm{eff}}(s)=\bar{U}_{a b}(s)+\sum_{a>c>b} X_{a b}^{(c)}(s) .
\end{aligned}
$$

If we use $U_{a b}^{\text {eff }}(s)$ and $\bar{U}_{a b}^{\text {eff }}(s)$ instead of $U_{a b}(s)$ and $\bar{U}_{a b}(s)$ in the first two lines of Eq. (65) the very last term in Eq. (65) can be dropped. We can combine our results into

$$
\begin{aligned}
\hat{N}_{a b}(s)= & U_{a b}^{\mathrm{eff}}(s)+\sum_{c, d} \int \frac{d \bar{s}}{\pi} \frac{s-\mu^{2}}{\bar{s}-\mu^{2}} \frac{\hat{N}_{a c}(\bar{s})}{\bar{s}-s} \hat{\rho}_{c d}(\bar{s}) \\
& \times\left[U_{d b}^{\mathrm{eff}}(\bar{s})-U_{d b}^{\mathrm{eff}}(s)\right] \\
& -\sum_{c, d} \int \frac{d \bar{s}}{\pi} \frac{s-\mu^{2}}{\bar{s}-\mu^{2}} \frac{D_{a c}\left(\bar{s}+i \epsilon^{\prime}\right) \bar{\rho}_{c d}^{L}(\bar{s})}{\bar{s}-s} \\
& \times\left[\bar{U}_{d b}^{\mathrm{eff}}(\bar{s})-\bar{U}_{d b}^{\mathrm{eff}}(s)\right] \\
& -\sum_{c} \int \frac{d \bar{s}}{\pi} \frac{\mu^{2}}{\bar{s}-\mu^{2}} \frac{\hat{N}_{a c}(\bar{s}) \rho_{c b}^{R}(\bar{s})}{\bar{s}-s+i \epsilon^{\prime}} .
\end{aligned}
$$

With Eq. (69) we derived a convenient basis for the derivation of a suitable integral equation to numerically solve for $\hat{\varsigma}_{a b}(s)$. After a few more steps we find

$$
\hat{N}_{a b}(s)=\hat{U}_{a b}(s)+\sum_{c, d} \int \frac{d \bar{s} s-\mu^{2}}{\pi} \hat{N}_{a c}(\bar{s}) \hat{\rho}_{c d}(\bar{s}) \hat{K}_{d b}(\bar{s}, s),
$$

with a well-behaved integral kernel $\hat{K}_{d b}(\bar{s}, s)$ and potential term $\hat{U}_{a b}(s)$. However, we first need to address the necessary analytic continuation of the $\hat{N}_{a b}(s)$ functions below $s=\left(m_{b}+M_{b}\right)^{2}$. It is argued that Eq. (69) can be used for that purpose. On general grounds we expect the $\hat{N}_{a b}(s)$ functions to inherit the cut lines of the generalized potential. The process of taking the limit $\epsilon \rightarrow 0$ of all contour lines, can be visualized as a twostep procedure. We first deform all lines in Fig. 3 such that they are in an $\epsilon^{\prime} \ll \epsilon$ vicinity of the three parallel lines passing trough the points $s=0$ and $s= \pm i \epsilon$. It is important that this is done without crossing any lines. Within this picture all horizontal cut lines of $\hat{N}_{a b}(s)$ sit on the two parallel lines that pass through $s= \pm i \epsilon$. In addition there are vertical cut lines. However, the contribution of the latter will be negligible in the limit $\epsilon \rightarrow 0$. In fact such contributions were already dropped in our derivations. In the absence of such vertical cut lines and at a still finite $\epsilon$ the functions $\hat{N}_{a b}(s)$ are analytic in the strip defined by $|\operatorname{Im} s|<\epsilon$. Given this picture it is evident that Eq. (69) provides the desired analytic continuation for $\hat{N}_{a b}(s)$. The integrals in Eq. (69) only generate cut lines that are on the $\pm i \epsilon$ lines.

The auxiliary functions $\hat{U}_{a b}(s)$ are presented in Fig. 14 as derived from our model (15). The complex functions are shown in the domain $s>\mu_{b}^{2}$ or $s>\left(m_{b}+M_{b}\right)^{2}$ only as needed for the evaluation of the functions $\hat{N}_{a b}(s)$ as shown in Fig. 13. Note that the functions $\hat{U}_{a b}(s)$ are piecewise continuous in the domain shown with the only discontinuous behavior being at the return point $s=\hat{\mu}_{b}^{2}$.

The derivation of the anticipated integral equation (70) is done with the help of the Green's functions $L(x, y)$ and $R(x, y)$. While we already introduced the "left" Green's function in Eq. (40), the "right" counterpart is readily identified with

$$
\begin{aligned}
\int d y\left[\delta(x-y)+\frac{1}{\pi} \frac{\rho^{R}(x)}{x-y+i \epsilon^{\prime}}\right] R(y, z)=\delta(x-z), \\
R(x, y)=\delta(x-y)-\frac{1}{\pi} \frac{\rho^{R}(x)}{x-y+i \epsilon^{\prime}} \\
+\sum_{n=1}^{3} \rho^{R}(x) \frac{U_{n}^{R}(x)-U_{n}^{R}(y)}{x-y} \frac{1}{\pi} u_{n}^{R}(y),
\end{aligned}
$$

where we emphasize that each of the objects $u_{n}^{R}(x)$ and $U_{n}^{R}(x)$ has a coupled-channel matrix structure at any $n=1,2,3$. The formal expressions for $u_{n}^{R}(x)$ and $U_{n}^{R}(x)$ can be extracted from our previous expression for $u_{n}^{L}(x)$ and $U_{n}^{L}(x)$ in Eq. (44). The similarity between the two Green's functions (left and right) is a consequence of the identity

$$
\rho_{a b}^{R}(x)=\rho_{b a}^{L}(x),
$$

which is reflected in Eqs. (37) and (66). This implies that if we evaluate $u_{n}^{L}(x)$ and $U_{n}^{L}(x)$ in Eq. (44) at $\bar{\rho}_{a b}^{L}(s)=\rho_{a b}^{L}(s)$ the desired objects follow with

$$
u_{n}^{R}(x)=\left[u_{n}^{L}(x)\right]^{\dagger}, \quad U_{n}^{R}(x)=\left[U_{n}^{L}(x)\right]^{\dagger} .
$$

It remains to recall our previous result (41) and apply the right Green's function from the right side in Eq. (69). We multiply the equation by $R(s, x)$ and integrate over $s$. This leads to the identification of the potential term $\hat{U}_{a b}(s)$ and the integral kernel $\hat{K}_{a b}(\bar{s}, s)$ of the following form: 


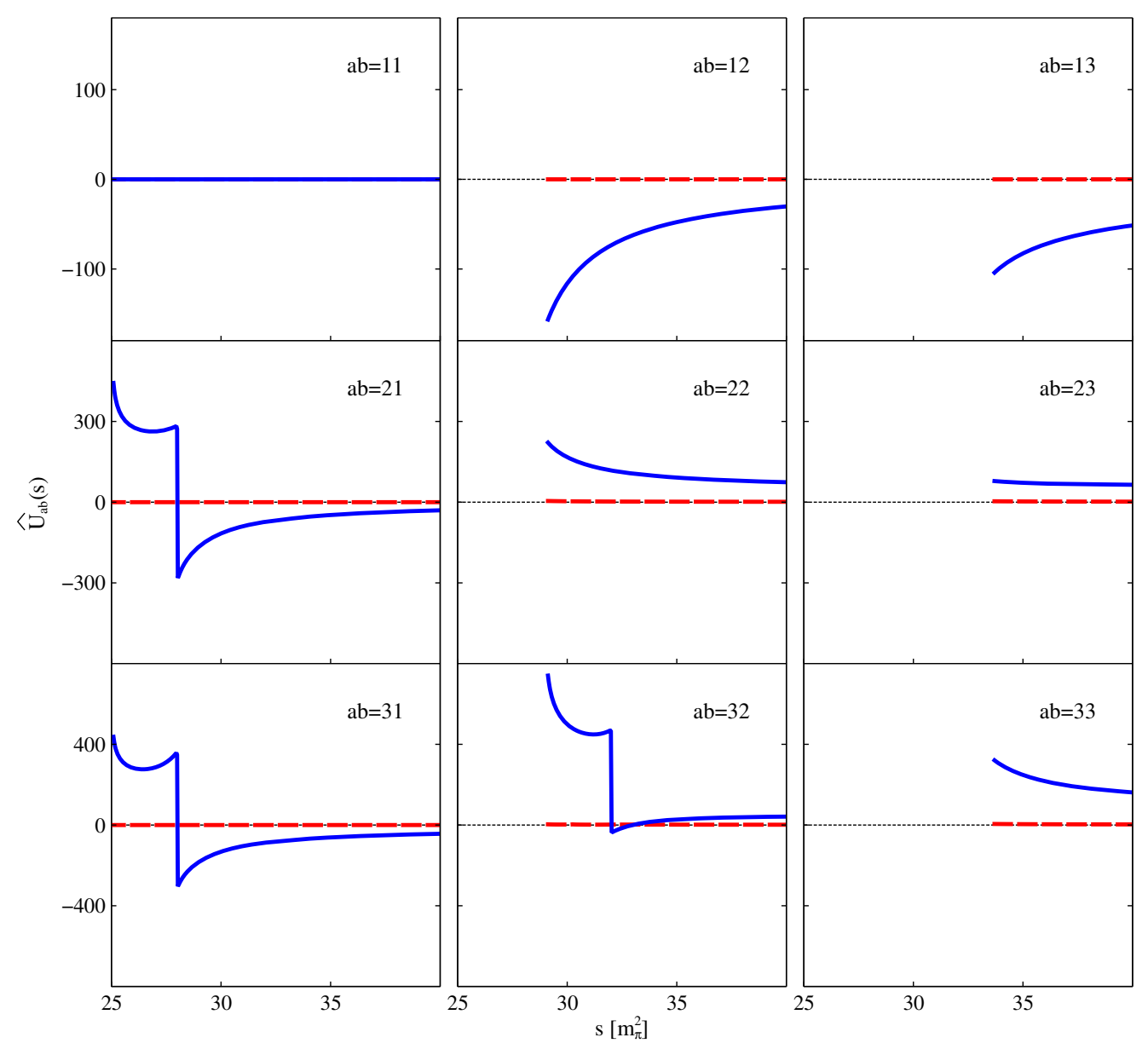

FIG. 14. The functions $\hat{U}_{a b}(s)$ of Eqs. (70) (74), and (77) for $s>\mu_{b}^{2}$ or $s>\left(m_{b}+M_{b}\right)^{2}$ in our schematic model (15). Real and imaginary parts are shown with solid blue and dashed red lines respectively.

$$
\begin{aligned}
\hat{U}_{a b}(s) & =\sum_{c} \int d \bar{s} \frac{s-\mu^{2}}{\bar{s}-\mu^{2}}\left[U_{a c}^{\mathrm{eff}}(\bar{s}) R_{c b}(\bar{s}, s)-\frac{1}{\pi} \bar{\rho}_{a c}^{L}(\bar{s}) \bar{K}_{c b}(\bar{s}, s)\right], \\
\bar{K}_{a b}(\bar{s}, s) & =\sum_{c, d} \int d x \int d y \int L_{a c}(\bar{s}, x) \frac{\bar{U}_{c d}^{\mathrm{eff}}(x)-\bar{U}_{c d}^{\mathrm{eff}}(y)}{x-y} R_{d b}(y, s), \\
\hat{K}_{a b}(\bar{s}, s) & =\bar{K}_{a b}(\bar{s}, s)+\int d y \frac{U_{c d}^{\mathrm{eff}}(\bar{s})-U_{c d}^{\mathrm{eff}}(y)}{\bar{s}-y} R_{d b}(y, s)-\int d y \frac{\bar{U}_{c d}^{\mathrm{eff}}(\bar{s})-\bar{U}_{c d}^{\mathrm{eff}}(y)}{\bar{s}-y} R_{d b}(y, s),
\end{aligned}
$$

with which we finally identify the ingredients of Eq. (70) in terms of the phase-space matrix $\hat{\rho}_{a b}(s)$ and the generalized potential $U_{a b}^{\text {eff }}(s)$ introduced already in Eqs. (66) and (69).

It is useful to derive somewhat more explicit expressions. This is readily achieved in terms of the identity

$$
\begin{aligned}
\int d z \frac{\bar{U}^{\mathrm{eff}}(x)-\bar{U}^{\mathrm{eff}}(z)}{x-z} R(z, y) & =\sum_{n=1}^{3} \frac{\bar{U}_{n}^{\mathrm{eff}}(x)-\bar{U}_{n}^{\mathrm{eff}}(y)}{x-y} u_{n}^{R}(y) \\
\bar{U}_{1}^{\mathrm{eff}}(x) & =\int \frac{d z}{\pi}\left[\Delta \bar{U}_{1}^{\mathrm{eff}}(z, x) U^{R}(z)+\Delta \bar{U}_{2}^{\mathrm{eff}}(z, x)\right] \frac{\rho^{R}(z)}{z-x} \\
\bar{U}_{2}^{\mathrm{eff}}(x) & =\bar{U}^{\mathrm{eff}}(x)-\int \frac{d z}{\pi}\left[\Delta \bar{U}_{1}^{\mathrm{eff}}(z, x) U^{R}(z)+\Delta \bar{U}_{2}^{\mathrm{eff}}(z, x)\right] \frac{\rho^{R}(z)}{z-x} U^{R}(z), \\
\bar{U}_{3}^{\mathrm{eff}}(x) & =\int \frac{d z}{\pi} \Delta \bar{U}_{1}^{\mathrm{eff}}(z, x) \frac{g^{R}(z) \rho^{R}(z)}{z-x}+\int \frac{d z}{\pi} \Delta \bar{U}_{2}^{\mathrm{eff}}(z, x) \frac{h^{R}(z) \rho^{R}(z)}{z-x}
\end{aligned}
$$


An analogous result holds for the action of the left Green's function $L(x, y)$ on such a structure. This can then be applied to derive the integral kernel

$$
\begin{aligned}
& \hat{K}(\bar{s}, s)=\sum_{m, n=1}^{3} u_{m}^{L}(\bar{s}) \frac{\hat{U}_{m n}(\bar{s})-\hat{U}_{m n}(s)}{\bar{s}-s} u_{n}^{R}(s), \\
& \hat{U}_{1 n}(x)=\int \frac{d z}{\pi} \frac{\rho^{L}(z)}{z-x}\left[U^{L}(z) \Delta \hat{U}_{1 n}(z, x)+\Delta \hat{U}_{2 n}(z, x)\right], \\
& \hat{U}_{2 n}(x)=\bar{U}_{n}^{\text {eff }}(x)-\int \frac{d z}{\pi} \frac{U^{L}(z) \rho^{L}(z)}{z-x}\left[U^{L}(z) \Delta \hat{U}_{1 n}(z, x)+\Delta \hat{U}_{2 n}(z, x)\right], \\
& \hat{U}_{3 n}(x)=\int \frac{d z \rho^{L}(z)}{\pi} \frac{\hat{u}^{L}}{z-x}\left[g^{L}(z) \Delta \hat{U}_{1 n}(z, x)+h^{L}(z) \Delta \hat{U}_{2 n}(z, x)\right]+\bar{U}_{n}^{\text {eff }}(x)-U_{n}^{\text {eff }}(x), \text { with } \Delta \hat{U}_{m n}(z, x)=\hat{U}_{m n}(z)-\hat{U}_{m n}(x),
\end{aligned}
$$

in terms of a set of analytic functions $\hat{U}_{m n}(s)$ and $u_{m}^{L}(s), u_{n}^{R}(s)$. We note that $g^{L}(x), h^{L}(x)$ were already introduced in Eqs. (44) and (75). The object $U_{n}^{\text {eff }}(x)$ is defined via Eq. (75) by replacing the source term with $\bar{U}^{\text {eff }}(x) \rightarrow U^{\text {eff }}(x)$. Using similar algebra leads to an explicit form for the potential term with

$$
\begin{aligned}
\hat{U}(s)= & U^{\mathrm{eff}}(s)-\int \frac{d \bar{s}}{\pi} \frac{s-\mu^{2}}{\bar{s}-\mu^{2}} \frac{U^{\mathrm{eff}}(\bar{s}) \rho^{R}(\bar{s})}{\bar{s}-s+i \epsilon^{\prime}}+\sum_{n=1}^{3} \int \frac{d \bar{s}}{\pi} \frac{s-\mu^{2}}{\bar{s}-\mu^{2}}\left\{U^{\mathrm{eff}}(\bar{s}) \rho^{R}(\bar{s}) \frac{U_{n}^{R}(\bar{s})-U_{n}^{R}(s)}{\bar{s}-s}\right. \\
& \left.-\sum_{m=1}^{3} \rho^{L}(\bar{s}) u_{a}^{L}(\bar{s}) \frac{\hat{U}_{m n}(\bar{s})-\hat{U}_{m n}(s)}{\bar{s}-s}\right\} u_{n}^{R}(s) .
\end{aligned}
$$

Altogether the linear integral equation (70) is cast into the simple form

$$
\hat{N}(s)=\hat{U}(s)+\sum_{m, n=1}^{3} \int \frac{d \bar{s} s-\mu^{2}}{\pi} \hat{\bar{s}-\mu^{2}} \hat{N}(\bar{s}) \hat{\rho}(\bar{s}) u_{m}^{L}(\bar{s}) \frac{\hat{U}_{m n}(\bar{s})-\hat{U}_{m n}(s)}{\bar{s}-s} u_{n}^{R}(s),
$$

where with Eq. (66) we finally arrive at the anticipated result (54).

We illustrate the role of the auxiliary matrices $\hat{U}_{m n}(s)$ as derived from our model (15). Since there are altogether 81 functions, we focus on the particular combinations

$$
\begin{aligned}
& H_{n}^{L}(s)=\sum_{m=1}^{3} u_{m}^{L}(s) \hat{U}_{m n}(s) \quad \text { and } \\
& H_{n}^{R}(s)=\sum_{m=1}^{3} \hat{U}_{n m}(s) u_{m}^{R}(s)
\end{aligned}
$$

as they are the relevant entities in Eq. (78). In Figs. 15-17 we show that each of the 27 elements of $H_{n, a b}^{R}(s)$ are piecewise continuous for $s>\mu_{b}^{2}$ or $s>\left(m_{b}+M_{b}\right)^{2}$. The complex functions are shown in the domain $s>\mu_{b}^{2}$ or $s>$ $\left(m_{b}+M_{b}\right)^{2}$ only as needed for the evaluation of the functions $\hat{N}_{a b}(s)$ as shown in Fig. 13. Note that $H_{n, a b}^{R}(s)$ are discontinuous only at the return points $s=\hat{\mu}_{b}^{2}$. The analogous property holds for the functions $H_{n, a b}^{L}(s)$ at $s>\left(m_{a}+M_{a}\right)^{2}$.
We should briefly summarize the general procedure to derive the phase shifts and inelasticity parameters for a given model interaction. Given the driving terms $\hat{U}(s)$ and $\hat{U}_{n m}(s)$ together with $u_{n}^{L}(s)$ and $u_{n}^{R}(s)$ as specified in Eqs. (44), (73), and (74)-(77) we use the linear set of equations (54) to determine the function $\hat{\varsigma}_{a b}(s)$ for $s>\left(m_{b}+M_{b}\right)^{2}$. This is a numerically stable task since all driving terms in Eq. (54) are sufficiently regular in the needed domain. In the next step we can compute the functions $D_{a b}(s+i \epsilon)$ in application of Eqs. (41)-(44). The functions $B_{a b}(s+i \epsilon)$ are evaluated from Eq. (59), which, however, requires the knowledge of the functions $\hat{\varsigma}_{a b}^{+}(s)$. This proceeds in two steps. First, given the functions $\hat{\varsigma}_{a b}(s)$ we can compute $\hat{N}_{a b}(s)$ from Eq. (78) at subthreshold energies $\mu_{b}^{2}<s<\left(m_{b}+M_{b}\right)^{2}$. Then with

$$
\hat{\boldsymbol{\varsigma}}_{a b}^{+}(s)=-\sum_{c} \hat{N}_{a c}(s) \rho_{c b}(s),
$$

the desired object $\hat{\zeta}_{a b}^{+}(s)$ is available and we can finalize the derivation of the functions $B_{a b}(s+i \epsilon)$. In turn the reaction amplitudes $T_{a b}(s+i \epsilon)$ can be reconstructed from Eqs. (24) and (31) in terms of the functions $D_{a b}(s+i \epsilon), B_{a b}(s+i \epsilon)$ 


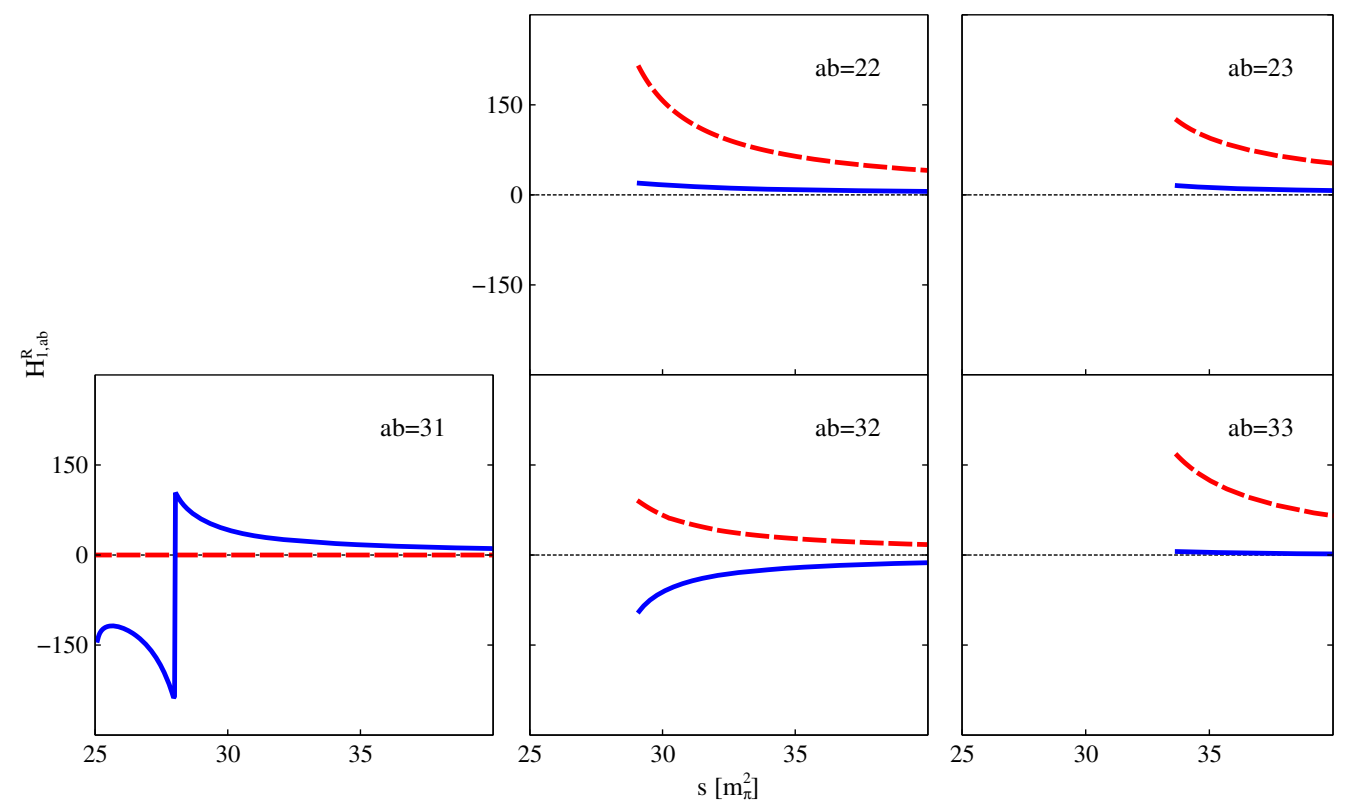

FIG. 15. The nonvanishing functions $H_{1, a b}^{R}(s)=\sum_{m=1}^{3} \hat{U}_{1 m}(s) u_{m}^{R}(s)$ for $s>\mu_{b}^{2}$ or $s>\left(m_{b}+M_{b}\right)^{2}$ in our schematic model (15). Real and imaginary parts are shown with solid blue and dashed red lines respectively.

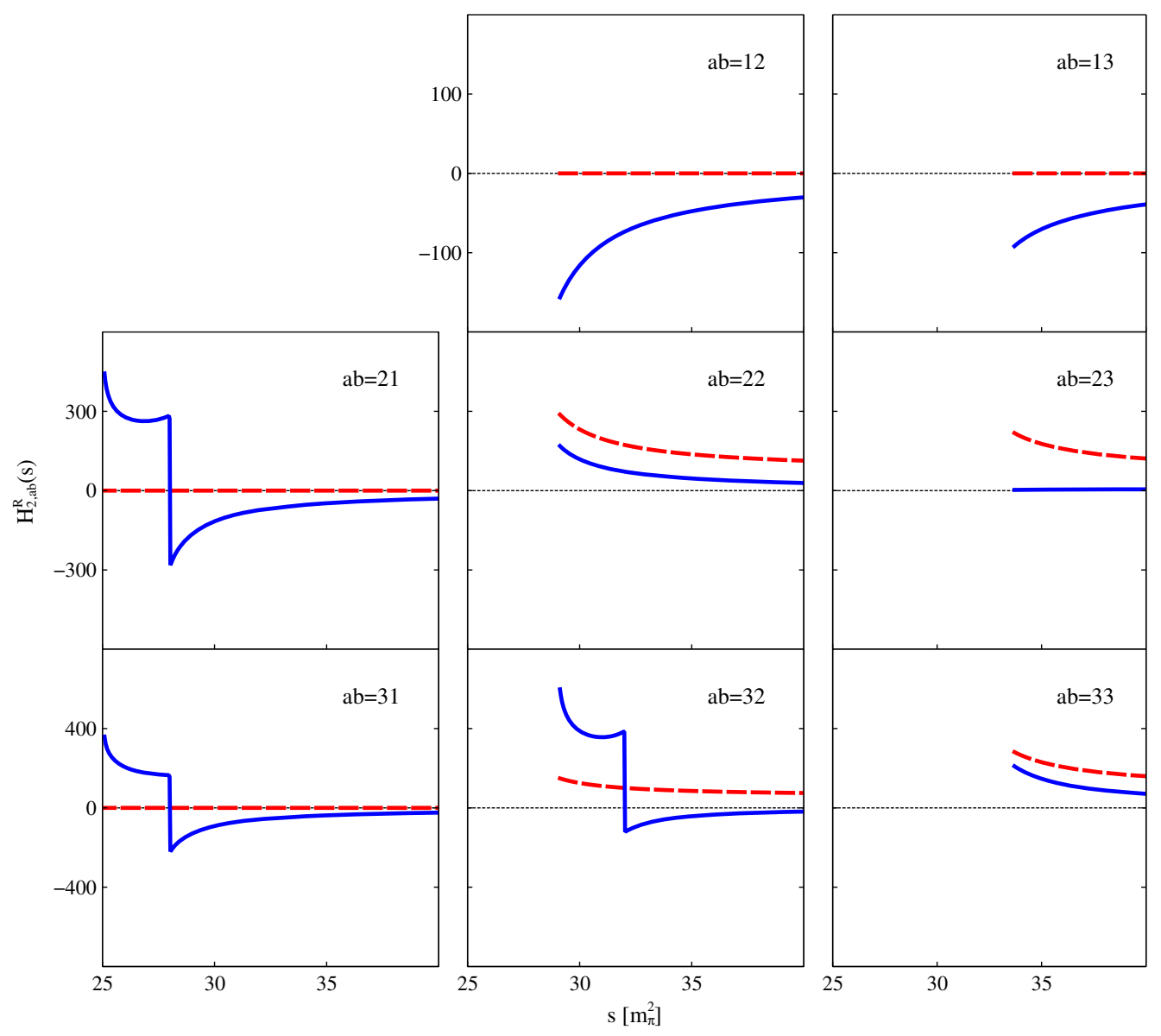

FIG. 16. The nonvanishing functions $H_{2, a b}^{R}(s)=\sum_{m=1}^{3} \hat{U}_{2 m}(s) u_{m}^{R}(s)$ for $s>\mu_{b}^{2}$ or $s>\left(m_{b}+M_{b}\right)^{2}$ in our schematic model (15). Real and imaginary parts are shown with solid blue and dashed red lines respectively. 


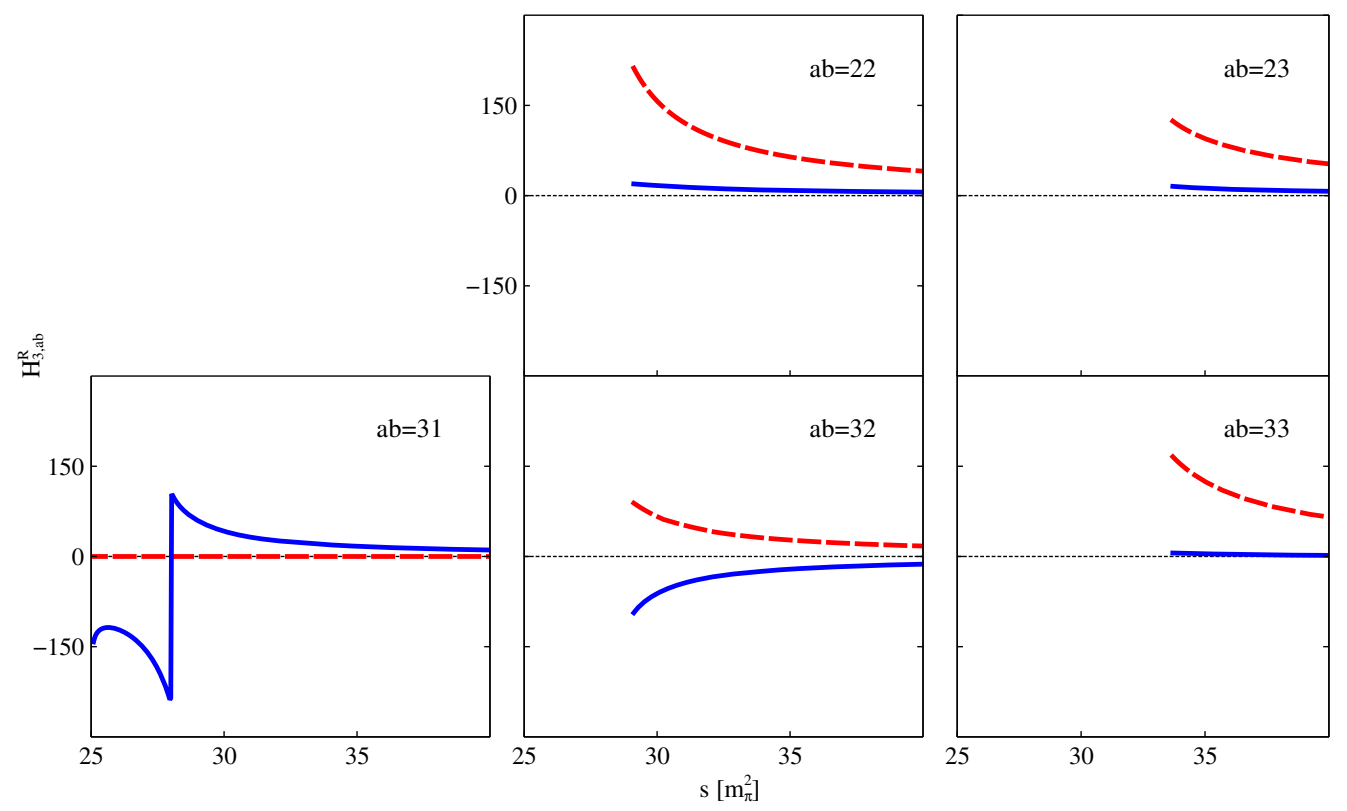

FIG. 17. The nonvanishing functions $H_{3, a b}^{R}(s)=\sum_{m=1}^{3} \hat{U}_{3 m}(s) u_{m}^{R}(s)$ for $s>\mu_{b}^{2}$ or $s>\left(m_{b}+M_{b}\right)^{2}$ in our schematic model (15). Real and imaginary parts are shown with solid blue and dashed red lines respectively.

and $U_{a b}(s+i \epsilon)$. The phase shifts and inelasticity parameters are then given by Eq. (16).

\section{SUMMARY AND OUTLOOK}

In this work we presented a novel framework to deal with coupled-channel systems in the presence of anomalous threshold effects. The framework was formulated for an arbitrary number of channels and is suitable for numerical simulations. We list the main cornerstones of our development.

(1) Given a generalized potential the coupled-channel reaction amplitudes were defined in terms of a set of nonlinear integral equations formulated on contours in the complex plane.

(2) The analytic structure of the generalized potential in the presence of anomalous threshold effects was clarified. The key observation was the fact that the latter must not satisfy the Schwarz reflection principle.

(3) We confirmed previous results that a physical approach must consider second-order terms in the generalized potential. The minimal contributions were identified with the terms that are odd under a Schwarz reflection.

(4) The nonlinear integral equation can be solved numerically by a suitable ansatz with Riemann integrals over real energies only. The specific form of the latter was derived for the first time. Explicit expressions for the driving terms were presented for an arbitrary number of channels.

(5) A schematic three-channel model was analyzed in the presence of anomalous thresholds. Throughout the development all key quantities were illustrated in this model. In particular the reaction amplitudes as well as the phase shifts and inelasticity parameters were computed and discussed.

Given our framework it is now possible to investigate coupled-channel systems including $J^{P}=1^{-}$and $J^{P}=\frac{3}{2}+$ states using realistic interactions. Such systems are notoriously challenging since a plethora of anomalous threshold effects are present. We expect such studies to shed more light on the possible relevance of the hadrogenesis conjecture, which predicts that such computations could generate a large part of the hadronic excitation spectrum in QCD.

\section{ACKNOWLEDGMENTS}

M. F. M. L. thanks E.E. Kolomeitsev for collaboration at an early stage of the project. C. L.K. was partially supported by the Országos Tudományos Kutatási Alapprogramok (OTKA, Hungary) fund K109462, the GSI theory group and the ExtreMe Matter Institute EMMI at the GSI Helmholtzzentrum für Schwerionenforschung, Darmstadt, Germany. 
[1] R. A. Briceno et al., Chin. Phys. C 40, 042001 (2016).

[2] C. A. Meyer and E. S. Swanson, Prog. Part. Nucl. Phys. 82, 21 (2015).

[3] M. F. M. Lutz et al., Nucl. Phys. A948, 93 (2016).

[4] M. R. Pennington, J. Phys. G 43, 054001 (2016).

[5] F. Zachariasen and C. Zemach, Phys. Rev. 128, 849 (1962).

[6] R. Dalitz, T. Wong, and G. Rajasekaran, Phys. Rev. 153, 1617 (1967).

[7] R. Logan and H. Wyld, Phys. Rev. 158, 1467 (1967).

[8] N. Kaiser, P. Siegel, and W. Weise, Nucl. Phys. A594, 325 (1995).

[9] J. A. Oller, E. Oset, and J. R. Pelaez, Phys. Rev. Lett. 80, 3452 (1998).

[10] J. A. Oller, E. Oset, and J. R. Pelaez, Phys. Rev. D 59, 074001 (1999).

[11] J. Nieves and E. R. Arriola, Nucl. Phys. A679, 57 (2000).

[12] A. Gomez Nicola and J. Pelaez, Phys. Rev. D 65, 054009 (2002).

[13] M. F. M. Lutz and E. E. Kolomeitsev, Nucl. Phys. A700, 193 (2002).

[14] M. F. M. Lutz, G. Wolf, and B. Friman, Nucl. Phys. A706, 431 (2002).

[15] J. Nieves and E. R. Arriola, Phys. Rev. D 64, 116008 (2001).

[16] E. E. Kolomeitsev and M. F. M. Lutz, Phys. Lett. B 585, 243 (2004).

[17] M. F. M. Lutz and E. E. Kolomeitsev, Nucl. Phys. A730, 392 (2004).

[18] C. Garcia-Recio, J. Nieves, E. R. Arriola, and M. J. V. Vacas, Phys. Rev. D 67, 076009 (2003).

[19] C. Garcia-Recio, M. F. M. Lutz, and J. Nieves, Phys. Lett. B 582, 49 (2004).

[20] A. M. Gasparyan and M. F. M. Lutz, Nucl. Phys. A848, 126 (2010).

[21] I. Danilkin, A. M. Gasparyan, and M. F. M. Lutz, Phys. Lett. B 697, 147 (2011).

[22] I. Danilkin, L. Gil, and M. F. M. Lutz, Phys. Lett. B 703, 504 (2011).

[23] A. Gasparyan, M. F. M. Lutz, and E. Epelbaum, Eur. Phys. J. A 49, 115 (2013).

[24] I. Danilkin, M. F. M. Lutz, S. Leupold, and C. Terschlusen, Eur. Phys. J. C 73, 2358 (2013).

[25] A. Gasparyan, M. F. M. Lutz, and B. Pasquini, Nucl. Phys. A866, 79 (2011).
[26] M. F. M. Lutz and I. Vidana, Eur. Phys. J. A 48, 124 (2012).

[27] Y. Heo and M. F. Lutz, Eur. Phys. J. A 50, 130 (2014).

[28] M. F. M. Lutz, E. E. Kolomeitsev, and C. L. Korpa, Phys. Rev. D 92, 016003 (2015).

[29] M. F. M. Lutz and E. E. Kolomeitsev, Found. Phys. 31, 1671 (2001).

[30] M. F. M. Lutz and E. E. Kolomeitsev, Nucl. Phys. A730, 110 (2004).

[31] E. E. Kolomeitsev and M. F. M. Lutz, Phys. Lett. B 582, 39 (2004).

[32] M. F. M. Lutz and M. Soyeur, Nucl. Phys. A813, 14 (2008).

[33] J. Hofmann and M. F. M. Lutz, Nucl. Phys. A763, 90 (2005).

[34] J. Hofmann and M. F. M. Lutz, Nucl. Phys. A776, 17 (2006).

[35] C. Terschlüsen, S. Leupold, and M. F. M. Lutz, Eur. Phys. J. A 48, 190 (2012).

[36] S. Mandelstam, Phys. Rev. Lett. 4, 84 (1960).

[37] J. S. Ball, W. R. Frazer, and M. Nauenberg, Phys. Rev. 128, 478 (1962).

[38] J. M. Greben and L. P. Kok, Phys. Rev. C 13, 489 (1976).

[39] X.-H. Liu, M. Oka, and Q. Zhao, Phys. Lett. B 753, 297 (2016).

[40] Z. Yang, Q. Wang, and U.-G. Meißner, Phys. Lett. B 767, 470 (2017).

[41] F.-K. Guo, U. G. Meißner, J. Nieves, and Z. Yang, Eur. Phys. J. A 52, 318 (2016).

[42] X.-H. Liu and G. Li, Eur. Phys. J. C 76, 455 (2016).

[43] J.-J. Xie and F.-K. Guo, Phys. Lett. B 774, 108 (2017).

[44] X.-H. Liu and U.-G. Meißner, Eur. Phys. J. C 77, 816 (2017).

[45] G. Chew and F. Low, Phys. Rev. 101, 1570 (1956).

[46] S. Mandelstam, Phys. Rev. 112, 1344 (1958).

[47] G. Chew and S. C. Frautschi, Phys. Rev. Lett. 7, 394 (1961).

[48] G. Frye and R. L. Warnock, Phys. Rev. 130, 478 (1963).

[49] J. Ball, R. Garg, and G. L. Shaw, Phys. Rev. 177, 2258 (1969).

[50] C. K. Chen, Phys. Rev. D 5, 1464 (1972).

[51] R. J. Eden, P. V. Landshoff, D. I. Olive, and J. C. Polkinghorne, The Analytic S-Matrix (Cambridge University Press, Cambridge, England, 1966).

[52] P. W. Johnson and R. L. Warnock, J. Math. Phys. 22, 385 (1981). 